\title{
Differential Effects of Dopaminergic Therapies on Dorsal and Ventral Striatum in Parkinson's Disease: Implications for Cognitive Function
}

\author{
Penny A. MacDonald ${ }^{1}$ and Oury Monchi ${ }^{2}$ \\ ${ }^{1}$ Department of Neurology \& Neurosurgery, McGill University, Montreal, QC, Canada \\ ${ }^{2}$ Unité de neuroimagerie fonctionnelle, Institut Universitaire de Gériatrie de Montréal, QC, Canada H3W 1 W5
}

Correspondence should be addressed to Penny A. MacDonald, penny.macdonald@gmail.com

Received 21 November 2010; Accepted 7 January 2011

Academic Editor: Antonio Strafella

Copyright ( $) 2011$ P. A. MacDonald and O. Monchi. This is an open access article distributed under the Creative Commons Attribution License, which permits unrestricted use, distribution, and reproduction in any medium, provided the original work is properly cited.

\begin{abstract}
Cognitive abnormalities are a feature of Parkinson's disease (PD). Unlike motor symptoms that are clearly improved by dopaminergic therapy, the effect of dopamine replacement on cognition seems paradoxical. Some cognitive functions are improved whereas others are unaltered or even hindered. Our aim was to understand the effect of dopamine replacement therapy on various aspects of cognition. Whereas dorsal striatum receives dopamine input from the substantia nigra (SN), ventral striatum is innervated by dopamine-producing cells in the ventral tegmental area (VTA). In PD, degeneration of SN is substantially greater than cell loss in VTA and hence dopamine-deficiency is significantly greater in dorsal compared to ventral striatum. We suggest that dopamine supplementation improves functions mediated by dorsal striatum and impairs, or heightens to a pathological degree, operations ascribed to ventral striatum. We consider the extant literature in light of this principle. We also survey the effect of dopamine replacement on functional neuroimaging in PD relating the findings to this framework. This paper highlights the fact that currently, titration of therapy in PD is geared to optimizing dorsal striatum-mediated motor symptoms, at the expense of ventral striatum operations. Increased awareness of contrasting effects of dopamine replacement on dorsal versus ventral striatum functions will lead clinicians to survey a broader range of symptoms in determining optimal therapy, taking into account both those aspects of cognition that will be helped versus those that will be hindered by dopaminergic treatment.
\end{abstract}

\section{Introduction}

Parkinson's disease (PD) is a neurodegenerative illness with prominent motor symptoms of tremor, bradykinesia, and rigidity. These motor symptoms result from degeneration of the dopamine-producing cells of the substantia nigra, leading to dopamine deficiency and dysfunction in the dorsal striatum. Cognitive dysfunction has long been recognized as a feature of Parkinson's disease with 20-50\% meeting criteria for dementia [1-5] and a far greater proportion displaying features of milder cognitive dysfunction [6]. Unlike the relatively clear-cut explanation for motor symptoms, debate has surrounded the locus of these cognitive impairments in PD. While initial explanations focused on cortical degeneration, which also occurs in $\mathrm{PD}$, particularly at later disease stages, studies have repeatedly failed to demonstrate correlation between cortical Lewy body dispersion and severity of cognitive impairment [7-11]. Studies in patients with basal ganglia lesions and investigations of cognition in healthy volunteers using neuroimaging are increasingly attributing cognitive functions to basal ganglia [12-17]. Some pathological studies confirm cognitive impairment in PD patients even in the absence of cortical compromise [18, 19]. Taken together, basal ganglia pathology and biochemical deficit might be an important cause for cognitive impairment in PD. Further complicating our understanding of cognitive function in PD, whereas the motor manifestations are clearly improved by dopamine replacement medications such as L3,4-dihydroxyphenylalanine (L-dopa) or dopamine receptor agonists, the effect of dopamine replacement therapy on 
cognition seems paradoxical. Some cognitive functions are improved by dopaminergic therapy whereas others are unaltered or even hindered. Our main aim, in fact, is to review and understand the effect of dopamine replacement therapy on different aspects of cognition, relating these findings to functions of different segments of basal ganglia.

Previous investigations suggest that individual segments of the basal ganglia mediate different elements of cognition. One approach for subdividing the striatum involves distinguishing the ventral striatum, comprising the nucleus accumbens and the most ventral portions of caudate and putamen, from the dorsal striatum, entailing the bulk of the caudate nuclei and putamen [20-22]. This distinction is important in $\mathrm{PD}$ given that the dopamine input to these regions are divergent and degenerate at different times and to varying degrees in disease evolution. Whereas dorsal striatum, responsible for the prominent motor symptoms, receives dopamine input from the substantia nigra (SN), ventral striatum is innervated by dopamine-producing cells in the ventral tegmental area (VTA). In PD, the VTA is significantly less affected than the $\mathrm{SN}$ at clinical disease onset and a disparity is maintained throughout the disease course [2326]. Given these differences, functions performed by dorsal striatum should improve disproportionately with dopamine replacement therapy compared to those subserved by ventral striatum. In fact, there is evidence that ventral striatum functions worsen with provision of dopaminergic therapy $[13,27-32]$. An explanation offered for this medicationinduced impairment is that these less dopamine-depleted brain regions are effectively overdosed by dopaminergic medications that are titrated to dorsal striatum-mediated motor symptoms [13, 28, 29, 32].

A central objective of this paper is therefore to define the different cognitive processes mediated by the more dopamine-depleted dorsal compared to the relatively spared ventral striatum, with the aim of providing a framework for predicting and understanding those cognitive processes that might be enhanced compared to those that will be hindered by dopaminergic therapy, at least at the early stages of the disease. Albeit somewhat simplified in that it does not address the impact of, nor incorporate findings related to, other VTA-innervated regions, such as prefrontal and limbic cortex, we will show that this approach accommodates and explains an impressive array of cognitive and neuroimaging findings in PD, providing a possible principle to predict and understand the effect of dopamine replacement therapy on cognition in this disease.

We will first present subtle cytoarchitectonic distinctions between dorsal versus ventral striatum, as well as the divergent regions to which they are reciprocally connected, as evidence of how these regions are differentially adapted to separate cognitive functions. We will next review the effect of dorsal versus ventral striatum lesions on cognition, as well as the cognitive functions that implicate dorsal versus ventral striatum in neuroimaging studies. As a test of the framework adopted here, that dorsal striatum functions are improved whereas ventral striatum functions are worsened by dopamine replacement, we will next compare those cognitive functions that are known to be improved versus those that are impaired by dopamine replacement therapy in PD patients to the pattern predicted by the lesion and neuroimaging studies. Table 1 summarizes these findings. Finally, we will survey the results of neuroimaging studies in PD patients on and off dopamine replacement therapy. These studies provide direct evidence of the effect of dopamine supplementation on brain activity in PD. Further, they provide an additional test of the hypothesis that variable effects of dopamine treatment in PD on distinct cognitive processes relate to their differential reliance on dorsal and ventral striatum.

1.1. Dorsal Striatum. In the dorsal striatum there are denser dopamine inputs and more numerous dendrites and spines on medium spiny neurons (MSNs) resulting in rapid and maximal dopamine stimulation through a wide range of input firing frequency and intensity [22, 33]. Due to high concentration of dopamine transporter (DAT), which is responsible for synaptic dopamine reuptake and clearance, synaptic dopamine is rapidly cleared, yielding short dopamine stimulation durations [22]. Taken together, this precisely-timed, brief, and consistently maximal receptor stimulation adapts dorsal striatum for rapid, flexible, and more absolute or binary responding as might be needed in deciding between alternatives. Suggesting an important role in performance, the dorsal striatum is reciprocally connected to a number of effector brain regions such as frontal eye fields, dorsal and rostral premotor cortex, supplementary, and primary motor cortex. Dorsal striatum projections also arise from and lead to dorsolateral prefrontal, somatosensory, and parietal association cortices, regions involved in executive functions [34]. In addition to an extremely high degree of convergence in striatum, MSNs receive very few projections from each cortical neuron [3538]. Dorsal striatum is consequently ideally positioned to sum diverse influences on responding, with vast numbers of cortical neurons each making only small contributions, requiring a concordance among many inputs to influence the excitation status of a given MSN. In turn, through reciprocal connections, single MSNs affect numerous cortical neurons. In this way, dorsal striatum coordinates activity in disparate cortical regions. These characteristics would suggest that dorsal striatum is ideally suited for selecting some stimuli or responses and suppressing others.

1.2. Ventral Striatum. Subtle cytoarchitectonic and neurochemical differences for ventral relative to dorsal striatum, such as smaller neuron size with fewer and more widelyspaced dendrites and spines, along with less significant dopaminergic input, have as a functional consequence that receptor stimulation with a single dopamine pulse is slower, and of lower and more variable intensity than in dorsal striatum [22]. This translates to greater differences comparing tonic versus phasic dopamine stimulation in ventral striatum, a fact demonstrated experimentally by Zhang et al. [33] who found nearly maximal dorsal striatum stimulation at even the lowest intensity and frequency dopamine impulses compared to much more graded, incremental responses in the ventral striatum. Owing to lower DAT concentration, 
TABLE 1: Cognitive functions that are enhanced, unchanged, or impaired by dopaminergic therapy, grouped according to their association with dorsal striatum, ventral striatum, or other brain regions.

\begin{tabular}{|c|c|c|c|}
\hline & Enhanced by dopaminergic therapy & $\begin{array}{l}\text { Unchanged by dopaminergic } \\
\text { therapy }\end{array}$ & Impaired by dopaminergic therapy \\
\hline \multirow{3}{*}{ Ventral striatum } & *Motivation & & Implicit and explicit learning \\
\hline & *Impulsivity & & Reversal learning \\
\hline & & & Orienting to stimuli \\
\hline \multirow{9}{*}{ Dorsal striatum } & Selective attention & Complex planning & Time estimation \\
\hline & Selective responding & Set shifting & \\
\hline & Complex planning & Task switching & \\
\hline & Category judgements & & \\
\hline & Time estimation & & \\
\hline & Visuospatial processing & & \\
\hline & Explicit and implicit retrieval & & \\
\hline & Set shifting & & \\
\hline & Task switching & & \\
\hline \multirow{3}{*}{$\begin{array}{l}\text { Other brain } \\
\text { regions }\end{array}$} & Spatial working memory & Nonspatial working memory & Simple reaction time \\
\hline & $\begin{array}{l}\text { Manipulating contents of working } \\
\text { memory }\end{array}$ & Set shifting & $\begin{array}{l}\text { Production of self-generated } \\
\text { sequences }\end{array}$ \\
\hline & & Task switching & $\begin{array}{l}\text { Generation of alternate uses of } \\
\text { common objects }\end{array}$ \\
\hline
\end{tabular}

* Are enhanced to a pathological degree.

dopamine stimulation is also of longer duration in ventral compared to dorsal striatum [22]. Together, these characteristics make the ventral striatum well suited for associating stimuli or events, even across time, in a graded fashion as would be essential for probabilistic or associative learning and for binding events that are temporally coincident into episodes. The regions to which ventral striatum are reciprocally connected also suggest its involvement in encoding and associating salient features of the environment. The ventral striatum receives inputs from and projects to anterior cingulate, orbitofrontal, and anterior temporal cortices, as well as to hippocampus, insula, amygdala, and hypothalamus. Due to a high degree of convergence, with 10-20 000 cortical or limbic neurons projecting to a single medium spiny neuron in the striatum, representations in basal ganglia are highly sparse relative to corresponding representations in cortex and limbic regions [35-38]. This degree of abstraction precludes storing of memory engrams within ventral striatum but given its significant reciprocal interconnectedness to multiple regions simultaneously, ventral striatum receives information about (a) top-down, goal-directed attentional biases, (b) bottom-up object and event salience, (c) multimodal representations of objects and events, and (d) the current motivational state of the organism [20]. Given persistence of stimulation, ventral striatum can further incorporate response outcome and reward information. Because connections to these diverse cortical and limbic regions are reciprocal, ventral striatum is in a position to harmonize activity in these distant brain regions as is needed for associating disparate, temporally coincident features into episodes. These anatomical characteristics suggest that ventral striatum could play an important role in learning and encoding.

\section{Cognitive Testing (a) in Patients with Basal Ganglia Lesions and (b) Using Neuroimaging in Healthy Volunteers}

2.1. Dorsal Striatum. Lesions in the dorsal striatum have been shown to impair set shifting and task switching (e.g., [39-42] but see [43-46]), category judgements [41], and suppression of irrelevant information and responses, particularly when the ignored stimulus is highly salient and the to-be-avoided response is over-learned (e.g., [39, 40, 44, $45]$ but see $[46,47])$. Patients with dorsal striatum lesions have been shown in one study to have deficits in reversal of previously acquired stimulus-reward relations [48]. Tests of planning (e.g., tower of Hanoi and porteus mazes [49]) and visuospatial processing [50-52] uncover deficits in patients with dorsal striatum lesions. A number of lesion studies have also revealed deficits in explicit (e.g., [39, 44, 49, 52, 53] but see $[46])$ and implicit memory $[53,54]$ but see $[55]$. Working memory $[39,41,44,48]$, language (e.g., $[39,56]$ but see $[43,57]$ ), word and face recognition (e.g., $[39,43]$ but see [58]), as well as explicit [53] and implicit learning (e.g., $[41,51,53,59-61]$ but see $[49,62])$, in contrast, tend to be spared.

Consistent with these lesions studies, shifting set and changing stimulus-reward or stimulus-response mappings (e.g., [63-66] but see [15]) are associated with increased activity in dorsal striatum. Responding to less well learned dimensions such as colour versus word in the Stroop task [12] or with less-practiced responses, as when pictures are named in a second language relative to a first language [67], also preferentially activate dorsal striatum. In a similar vein, dorsal striatum is more engaged when responding to a target 
location that was inaccurately predicted by a cue when cues had previously been predictive of the correct target location [68]. That is, dorsal striatum is engaged when previously informative stimuli must now be disregarded.

Dorsal striatum is preferentially activated for learned relative to random motor sequences [69], for familiar items in an episodic recognition test [70], during category judgements $[17,71]$, especially when there is significant category uncertainty [72], for rewarded relative to unrewarded stimuli $[17,73]$ and responses [74, 75], and in distinguishing and estimating different time durations [7685]. Dorsal striatum activity remains significantly increased above baseline throughout these experiments, well after sequences, categorization rules, or stimulus-reward and response-reward relations have been acquired, suggesting that dorsal striatum is involved in performance rather than learning. Tests of visuospatial processing, such as mental rotation, further implicate dorsal striatum in $\mathrm{AMRI}$, although significant increases in activity were noted for male subjects only [86].

Dorsal striatum has been associated with risk aversion during decision making [87] although preferential activation has been noted when speed is emphasized over accuracy in a motion judgement task [88]. Recent findings implicated dorsal striatum in encoding the joint dimensions of reward magnitude and subjective value (i.e., marginal utility) as well as temporal discounting, supporting a role for the dorsal striatum in integrating divergent influences on decision making $[89,90]$.

2.2. Ventral Striatum. There have been very few examinations of cognition following lesions circumscribed to the ventral striatum, mostly owing to the rarity of such small and strategically placed lesions. In human participants, Goldenberg and colleagues [91] reported a case of anterograde amnesia for verbal material following a left nucleus accumbens bleed. Despite an inability to learn new verbal material whether testing memory with recall or recognition, this patient performed normally on tests of retrospective verbal memory, divided and shifting attention, Wisconsin card sorting (WCST), tower of London (TOL), working memory, language, encoding and retrieval of nonverbal information. Taken together, this pattern of deficits and spared functions suggests a critical role for the ventral striatum in encoding associations, with left ventral striatum lateralized for language. Calder and colleagues [92] revealed anger recognition deficits in 3 patients with left and 1 patient with right ventral striatum lesions despite otherwise normal visual processing. Martinaud and colleagues [93] found that left ventral striatum lesions, following anterior communicating artery aneurysm, were associated with behavioural deficits, reduced daily activities, and hyperactivity. Finally, Bellebaum and colleagues [48] looked at acquisition and reversal of stimulus-reward associations in 3 patients with isolated ventral basal ganglia lesions compared to patients with dorsal or dorsal plus ventral basal ganglia lesions as well as to control participants. All patients, irrespective of group, displayed deficits in reversing previously acquired stimulusreward contingencies. There were no specific or consistent patterns noted for patients with ventral basal ganglia lesions in their experiment, consequently.

Functional magnetic resonance imaging (fMRI) experiments have shown that the degree to which a motor sequence is implicitly learned correlates with ventral striatum activity [69] and that ventral striatum activity is greatest early in learning, and is preferential for positive feedback relative to negative feedback during initial learning [17, 74, 94, 95]. This ventral striatum-mediated, stimulus-reward learning occurs even without intention or consciousness [96]. Ventral striatum activity drops off as performance asymptotes [69]. Once learning is established, ventral striatum activity increases over baseline tasks only (a) for unexpected rewards delivered for previously unrewarded stimuli or when reward is omitted for previously rewarded items (i.e., prediction errors; [97102]), (b) for punishment after errors [103], or (c) in reversal learning experiments when criterion is reversed and selection of previously rewarded stimuli now elicits negative feedback $[17,65,104-106]$. Ventral striatum is differentially activated by salient [107-109], valued [107, 108, 110, 111], or novel stimuli $[17,112]$, and even for passively received monetary or social rewards [113]. Differential ventral striatum activity reflects both magnitude and probability of reward [114], as well as probability of a given outcome (e.g., [115-117] but see $[114]$ ). Taken together, the ventral striatum seems to be engaged when a stimulus or event in the environment signals the possibility of new learning.

Heightened ventral striatum activity has been shown in a number of studies to be associated with more impulsive choices $[118,119]$ and ventral striatum has not been implicated in response inhibition or response stopping [120, 121]. Ventral striatum activity is greater for riskier choices [118, $119,122,123$ ] and for more immediate rewards (i.e., temporal discounting; [124-126]). Further, negative functional interaction between nucleus accumbens and anteroventral prefrontal cortex was associated with decisions favouring long-term goals relative to an immediate reward [127]. That is, high levels of activity in nucleus accumbens correspond to lower anteroventral prefrontal cortex activity, which in turn was associated with decisions favouring immediate rewards over long-term objectives.

Consistent with findings in patients with ventral striatum lesions, neuroimaging studies have found that nucleus accumbens activity associates with encoding of facial emotional expressions [128]. Mühlberger and colleagues [129] further showed that ventral striatum activity was greater when control participants observed changes from angry to happy or neutral facial expressions. Finally, Liang and colleagues [130] found that ventral striatum activity correlated with extremes of facial attractiveness.

2.3. Summary: Cognitive Testing (a) in Patients with Basal Ganglia Lesions and (b) Using Neuroimaging in Healthy Volunteers. The dorsal striatum is implicated in selecting among various stimuli and competing responses, when divergent influences impinge on decision making and particularly when selection requires discounting more salient stimuli or overriding prepotent responses. Dorsal striatum is involved in complex planning tasks and in distinguishing among 
groups of stimuli and responses, tracking whether an item belongs to one category over another, is rewarded versus unrewarded, or is familiar versus novel. The dorsal striatum is implicated in time discrimination and estimation, as well as in visuospatial processing. From this review, we surmise that, whereas individual cortical regions might be specifically sensitive to separate aspects of a stimulus, situation, or event, such as salience, preference, motivational value, reward, speed, or accuracy, the dorsal striatum integrates all of these influences to yield an optimal, considered criterion, that maximizes and regulates accurate decision making, selective responding, and planning. Conversely, the dorsal striatum's necessity is significantly lessened for decisions that can be accomplished relying on a single dimension to guide behaviour-particularly if this dimension is most salient [15]. This could account for the inconsistent findings with respect to task- or set shifting deficits in patients with dorsal striatum lesions and for the occasional finding that these tasks do not preferentially activate dorsal striatum in neuroimaging investigations. An aim of future studies should be to better understand these inconsistencies.

In contrast, both lesion and neuroimaging studies suggest that the ventral striatum is extensively implicated in multiple aspects and forms of learning. Ventral striatum is involved in orienting attention to salient, novel, or valued stimuli and seems to mediate motivation, facilitating approach behaviours. Finally, some evidence suggests that ventral striatum might have a role in facial emotional processing. Both implicit and explicit learning and tests of implicit and explicit memory implicate ventral striatum. Unlike hippocampus and associated temporal cortex that seem specialized for encoding information when memory is subsequently explicitly probed, ventral striatum is implicated in more generalized encoding function. To our knowledge, no studies have examined this issue as a central aim and we are currently investigating this question.

\section{Effect of Dopamine Replacement Therapy on Cognition}

A number of studies have investigated the effects of dopamine replacement therapy on cognition in PD. At first blush, these results seem paradoxical. Whereas inconsistencies in this literature surely owe, at least in part, to differences in sample size, diverse methodologies, discrepancies in patient characteristics, such as age, disease duration and severity, PD-dominant side, and even genetic profile, we postulate that the differential reliance on the dorsal and ventral striatum of the cognitive function under investigation, accounts for most of this variability.

The dorsal striatum is significantly depleted of dopamine at all stages of clinical PD. The ventral striatum, in contrast, is substantially less dopamine deprived, especially early in the disease course. Because dopaminergic supplementation is titrated to dorsal striatum-mediated striatum motor functions, it is suggested that ventral striatum is overdosed and its functions are impaired whereas dorsal striatum becomes dopamine replete and operations that it mediates are improved. We test this explanation for the effect of dopaminergic therapy on cognitive functions in $\mathrm{PD}$, by relating (a) the pattern of cognitive improvements and impairments subsequent to dopamine replacement in $\mathrm{PD}$, to (b) the cognitive functions that seem attributed to the dorsal and ventral striatum outlined above.

\subsection{Cognitive Functions Improved by Dopamine Replacement} Therapy in PD. A number of studies have shown that administration of dopamine replacement therapy improves cognitive function in patients with PD. Impairments for PD patients in switching attention from one stimulus dimension [131-135] but see [29], or one response to another [134, 136], as well as in selecting between alternatives with high response conflict [137] are redressed by dopamine replacement. Similarly, although maintenance and retrieval of nonspatial information in working memory per se seems to be unaffected by dopaminergic therapy ([138-140] but see $[141,142])$, medication improves manipulation of the contents of working memory [138, 139]. Patients were impaired on a measure of verbal fluency compared with normal controls when tested off medication but there were no group differences on medication [29]. Remembering to perform an action at a specified time, so-called prospective remembering, was impaired in PD patients tested off but not on medication [143, 144]. Impairment in generating lines of varying lengths - an action planning deficit-in PD patients was improved with dopamine replacement whereas a deficit in repeatedly producing lines of only two lengths in the simple figure replication condition was not [145]. Also suggesting motor planning improvement with dopaminergic medication, PD patients on dopamine medication demonstrated better and normalized chunking of motor movements [146], despite normal sequence learning both off and on medication. In a similar vein, although learning simple stimulus-response relations was unaltered by dopamine replacement, chaining these learned associations to achieve a long-term goal was impaired in PD patients tested off medication. Chaining these events to achieve the end goal was improved when patients were tested on dopaminergic therapy [147]. Whether these results owe to a medication-remediable deficit in planning, learning, or retrieval, is unclear, however. Together, the findings surveyed above dopamine repletion improves cognitive flexibility, planning, and possibly long-term retrieval.

In contrast to nonspatial working memory, spatial working memory deficits have been shown to improve with dopaminergic treatments [148-151], perhaps related to improvement in visuospatial processing. Consistent with the latter interpretation, category-specific (i.e., animals) object recognition using degraded images has been shown to be compromised in de-novo $\mathrm{PD}$ patients relative to PD patients receiving dopaminergic therapy and healthy controls. This deficit was remediated with introduction of dopamine replacement medications [152].

Finally, a number of studies have demonstrated impaired behavioural performance on time estimation and motor timing tasks in PD patients relative to controls. These deficits improve with dopaminergic medication ([153-156] but see $[157,158])$. 
3.2. Cognitive Functions Unaffected by Dopamine Replacement Therapy in PD. Some studies have revealed no effect of dopamine replacement therapy on cognitive function. Shifting to a previously irrelevant dimension was impaired in $\mathrm{PD}$ patients relative to controls but was not improved by administration of L-dopa [27, 138, 139]. PD patients were impaired compared to controls in generating proper names but this impairment was not improved with dopamine replacement [159]. Nagano-Saito and colleagues [160] found no improvement on performance of the TOL on dopamine agonist relative to off medication.

\subsection{Cognitive Functions Impaired by Dopamine Replacement} Therapy in PD. Learning was most commonly impaired in PD patients tested on dopamine replacement therapy. A number of studies have revealed deficits after dopamine replacement in probabilistic associative learning, although PD patients off medication performed equivalently to controls [29, 140, 158]. Shohamy and colleagues [161] found that dopaminergic medication impaired learning of an incrementally acquired, concurrent discrimination task, whereas off medication PD patients performed as well as controls. Sequence learning was reduced for PD patients on medication [146, 162-164]. Dopamine supplementation in PD patients yielded reduced facilitation for consecutive, consistent stimulus-stimulus pairings in a selection task compared to normal implicit learning and hence facilitated responding when tested off medication [137]. Once stimulus-reward associations have been learned, reversing probabilities of stimulus-reward associations is also impaired for PD patients on dopamine replacement therapy [27, 32, 40, 104, 165-167]. Finally, dopamine therapy impaired learning from negative feedback [168].

Another frequent deficit in PD patients on dopamine replacement therapy is in impulse control [27, 169]. As an example, impulsive betting despite appropriate and deliberate decision making was noted following L-dopa administration in PD patients [132, 140]. L-dopa therapy in $\mathrm{PD}$ patients has been shown to increase the tendency to choose earlier relative to later rewards, regardless of reward magnitude (i.e., temporal discounting) compared to decision making on placebo. Dopaminergic therapy, particularly dopamine agonist use, in PD has clearly been shown to increase a number of impulse control disorders such as pathological gambling, compulsive sexual behaviour, compulsive buying, and binge eating $[170,171]$. The dopamine dysregulation syndrome in which PD patients overuse their dopamine replacement medications is a further example of enhanced motivation toward rewarding behaviours with therapy [172].

Simple reaction time was increased with administration of L-dopa [173] and apomorphine [174]. Time estimation in the seconds but not millisecond range was impaired in patients on relative to off medication and healthy controls $[155,175]$. Finally, impairment in generation tasks such as subject-ordered pointing [29] or production of alternate uses for common objects [167] have also been noted in PD patients on medication.
3.4. Effect of Dopamine Therapy in Healthy Controls. A number of studies have investigated the effect of dopaminergic modulation on cognitive function in healthy volunteers. Breitenstein and colleagues [176] found that administering a dopamine agonist significantly impaired novel word learning in healthy volunteers compared to placebo. Similarly, Pizzagalli and colleagues [177] and Santesso and colleagues [178] found that reward learning was impaired in healthy human volunteers after administering a single dose of pramipexole. Probabilistic reward learning relies on the ventral striatum $[74,94,96]$ and consequently these findings strengthen the contention that impaired learning in PD patients on medication results from overdose of VTA-innervated ventral striatum. Pine and colleagues [90] showed that in healthy controls administration of L-dopa increased temporal discounting in a decision making task, with more numerous smaller but sooner reward choices relative to larger but later reward options, compared to performance after receiving placebo or haloperidol. Schnider and colleagues [179] found that L-dopa, but not risperidone or placebo, increased false positive responses, without altering overall memory performance, in healthy volunteers tested in a memory paradigm that had previously been shown to be sensitive in confabulating patients. These findings suggest a less conservative response criterion compatible with increased impulsivity seen with dopamine replacement in healthy volunteers, paralleling findings in PD. Finally, Luciana and colleagues [180] found that bromocriptine, a dopamine agonist, facilitated spatial delayed but not immediate memory performance in healthy volunteers.

Conversely, others have investigated the effect of dopamine receptor antagonists on cognition in healthy volunteers with the aim of simulating the dopamine deficiency in PD. Set shifting impairments have been induced by this manipulation, consistent with performance of PD patients off medication [30]. Similarly, Nagano-Saito and colleagues [181] showed that after consuming a drink deficient in the dopamine precursors tyrosine and phenylalanine, post-set shift response times were increased in the WCST compared to when they performed the task, after consuming a drink balanced in amino acids. Finally, the effect of dopamine receptor antagonism on working memory in healthy controls has been inconsistent $[30,182,183]$.

\subsection{Summary: Effect of Dopamine Replacement Therapy on} Cognition. Based on our review, the pattern of improvements and impairments in PD patients following dopamine supplementation are well accounted for by differential baseline dopamine innervation of the dorsal and ventrals striatum, with very few exceptions. Consistent with conclusions about cognitive functions ascribed to dorsal striatum arising from lesion and neuroimaging studies, selecting among alternative stimuli and responses, particularly when there is high conflict or when enacting a decision requires disregarding previously relevant stimulus dimensions or responses, is improved by dopamine replacement in PD. Also consistent with lesion and neuroimaging studies, dopaminergic therapy remediates long-term memory retrieval, planning, visuo spatial processing, as well as time estimation and 
motor-timing deficits. Providing convergent evidence for dopamine's modulatory role in these executive functions, dopamine antagonists in healthy volunteers have been shown to impair set shifting.

Numerous studies reveal impaired learning in PD patients on relative to off dopamine replacement therapy as would be expected in reviewing lesion and neuroimaging studies of ventral striatum function. Impaired simple reaction time in $\mathrm{PD}$ patients on medication, which could owe to impaired orienting, also would not be inconsistent with functions ascribed to ventral striatum from our survey of lesion and neuroimaging studies. Further bolstering the dopamine overdose hypothesis to account for deterioration of some cognitive functions in medicated PD patients, dopaminergic therapy in healthy volunteers actually impairs learning, exactly paralleling the pattern observed in PD patients. To reiterate, the central contention of the dopamine overdose hypothesis is that because the VTA is relatively spared and hence ventral striatum dopamine is adequate, especially early in $\mathrm{PD}$, dopamine replacement, dosed to remediate the dorsal striatum-mediated motor symptoms, effectively causes an over-supply of dopamine to the ventral striatum, interfering with its function.

Not consistent with the view that dopamine overdose disrupts ventral striatum-mediated processes, increased impulsivity in PD patients on dopamine replacement actually suggests an enhancement of ventral striatum function. Lesion and imaging studies have shown that ventral striatum mediates motivation, approach behaviour, and impulsive choices. Also paralleling findings in PD, dopamine replacement in healthy volunteers increases impulsive choices and enhances false positive responses in a memory paradigm, consistent with greater impulsivity. While still in line with an account of ventral striatum dopamine over-supply, these findings cannot be explained by the claim that dopamine excess interferes with functions of ventral striatum. We submit that a possible explanation for opposing effects of dopamine replacement on these ventral striatum-mediated functions could owe to their differential reliance on phasic or relative, versus tonic or absolute dopamine receptor stimulation. In reviewing biological features of the ventral striatum, low tonic, with graded phasic dopamine responses, sensitive to frequency and degree of stimulation, are characteristics that render the ventral striatum particularly suited for encoding associations between stimuli, responses, outcomes, or events. If these graded dopamine signals convey strength of association then administration of bolus dopamine therapy could conceivably interfere with this encoding. Further, decreased DAT for clearing synaptic dopamine makes the ventral striatum even more vulnerable to disruption by bolus dopamine administration. In contrast, those functions of ventral striatum that depend on absolute dopaminergic tone and not upon extracting information from degree of dopamine receptor stimulation or from relative signal-to-noise ratio might be increased, albeit to a pathological level, by dopaminergic therapy. Impulsivity, an inclination to act prematurely without adequate consideration of relevant determinants of behaviour, might depend on absolute dopaminergic tone in the ventral striatum. Administration of dopaminergic therapy and consequent ventral striatum dopamine overdose might enhance this tendency to a detrimental degree.

Some studies have revealed no effect of dopamine replacement therapy on cognitive function. Possibly reflecting bias against publishing null results, there are far fewer examples of functions that are neither helped nor hindered by dopaminergic therapy in PD and hence a clear trend does not emerge. Given a variety of reasons for statistical equivalence, such as true equality between conditions and populations, inadequate power to detect differences, as well as a $20 \%$ Type 2 error rate compared to a more acceptable $5 \%$ Type 1 error rate, interpretation of null results can be problematic and should be done cautiously.

Remediable deficits in verbal fluency and in manipulating the contents of working memory with administration of dopaminergic therapy are not clearly predicted by the dorsal striatum lesion and neuroimaging studies. Further, time estimation has been attributed to dorsal striatum $[14,15,39-42]$ and therefore impairment in this process with dopamine replacement would not be explained by the simple framework applied here. Finally, decreased response generation with medication is not predicted by the lesion and neuroimaging literature reviewed here. These few inconsistencies might relate to effects of dopamine replacement on other brain regions, particularly those that also receive input from the relatively-spared VTA, such as prefrontal and limbic cortices, that we have not discussed in this review. Alternatively, a more complete understanding of the functions of the dorsal and ventral striatum might resolve these discrepancies. Overall, however, the framework adopted in this review accommodates a significant number of findings, despite the few inconsistencies encountered. Next, we review neuroimaging studies in PD patients on and off medication.

\section{Functional Neuroimaging in PD}

4.1. Neuroimaging in PD Patients off Dopaminergic Medication. Neuroimaging studies of patients with PD have revealed differences in regions of activation and de-activation at rest. A number of investigations have shown increased activity in the thalamus, globus pallidus, pons, and primary motor cortex compared to reductions in lateral premotor and posterior parietal areas [184]. Those patients who were not demented but performed abnormally on neuropsychological tests relative to controls additionally revealed reductions in medial prefrontal regions, dorsolateral prefrontal cortex, premotor cortex, rostral supplementary motor area, precuneus, and posterior parietal regions along with relative increases in cerebellar cortex and dentate nuclei [184].

Changes in patterns of activation are also described in PD patients off medication performing cognitive tasks. Investigating the effect of retrieval and manipulation of working memory contents on default mode network in PD patients off medication, van Eimeren and colleagues [185] found that PD patients only appropriately deactivated medial prefrontal cortex and in fact increased activation of precuneus and posterior cingulate cortex. The default network involves precuneus, medial prefrontal, posterior cingulate, lateral 
parietal, and medial temporal cortices and is characterized by deactivation during the performance of executive tasks in healthy volunteers $[186,187]$. Connectivity analysis also revealed that medial prefrontal cortex and the rostral ventromedial caudate nucleus were functionally disconnected in PD, further supporting disturbance of the default network in PD. Others have found that hypometabolism and decreased endogeneous dopamine in dorsal striatum, as measured by $\left[{ }^{18} \mathrm{~F}\right]$ DOPA PET, $\left[{ }^{11} \mathrm{C}\right]$-raclopride (RAC) PET, or fMRI, are directly correlated with poorer performance on the WCST and on tests of working, verbal, and visual memory in PD patients [188-190]. Schonberg and colleagues [191] further showed decreased prediction error signals in dorsal striatum in a reinforcement learning study using fMRI in PD relative to controls. Finally, dorsal-striatum-involving tasks (e.g., set-shifting under uncertainty in card sorting tasks) also reveal decreased activations in striatum-associated cortical regions such as posterior parietal regions, ventrolateral and dorsolateral prefrontal cortex when planning a set shift, as well as in premotor cortex during set-shift execution $[14,192]$.

In contrast, activity in the ventral striatum and cortical regions to which it is reciprocally connected, is comparable or, rarely, is enhanced in PD patients off medication compared to controls in neuroimaging studies. As previously noted, medial prefrontal cortical regions-reciprocally connected to ventral striatum and innervated by VTAappropriately deactivate during an executive task in PD patients off medication [185]. Prediction error signals in a reinforcement learning study using fMRI, were normal in the ventral striatum in PD patients tested off medication, despite impairments in the dorsal striatum. Sawamoto and colleagues [190] found that the same contrast of spatial working memory versus visuomotor processing that yielded hypometabolism in dorsal caudate for PD patients relative to controls, showed comparable between-group activity in anterior cingulate, a region reciprocally connected to the ventral striatum and receiving dopamine input from VTA. Finally, PD patients revealed increased activations relative to controls in prefrontal and posterior parietal cortex for cognitive processes that did not implicate caudate nucleus, such as in conditions that required neither planning nor executing a set shift in a card sorting task [14, 192]. This enhanced cortical activity was associated with poorer cognitive performance, however.

4.2. Summary: Neuroimaging Results in PD Patients off Dopaminergic Medication. Overall, these imaging studies are consistent with the notion that decreased cognitive performance in $\mathrm{PD}$ relates primarily to dopaminergic deficit and dysfunction of the dorsal striatum. Functional impairments owe to dorsal striatum dysfunction per se as well as to consequent deregulation of cortical networks involving dorsal striatum. These investigations further support that in undemented PD patients, ventral striatum and its cortical networks are unperturbed in the off state, which we attribute to preserved VTA dopaminergic function. On occasion, increased cortical activity is noted for $\mathrm{PD}$ patients off dopaminergic medications relative to controls, although this does not necessarily translate to improved cognitive performance $[14,149,192]$. Increased number and extent of some cortical regions recruited by $\mathrm{PD}$ patients while performing cognitive tasks off medication, could owe to aberrant up-regulation of regions that are normally opposed or inhibited by dorsal striatum and its cortical networks. Studies aimed specifically at contrasting dorsal versus ventral striatum-mediated cognitive functions in $\mathrm{PD}$ patients off medication relative to controls using neuroimaging are lacking. These studies are needed not only to directly assess the differential metabolic impairments of the dorsal and ventral striatum in PD but also to understand the impact, if any, of dorsal-striatum dysfunction on baseline ventral striatum metabolic function, independent of medication. The section that follows summarizes the effects of dopamine replacement in $\mathrm{PD}$ patients and dopamine modulation in healthy controls on patterns of brain activity assessed by functional neuroimaging.

\section{Effect of Dopamine Modulation on Brain Activity}

5.1. Normalization of Neuroimaging Patterns with Dopaminergic Therapy in PD Patients. In the resting state, $\mathrm{Wu}$ and colleagues [193] found that PD patients in the off state had significantly decreased functional connectivity between the supplementary motor area, left dorsolateral prefrontal cortex, and left putamen, along with increased functional connectivity among the left cerebellum, left primary motor cortex, and left parietal cortex compared to normal subjects. Administration of L-dopa normalized the pattern of functional connectivity in $\mathrm{PD}$ patients with degree of restoration correlating with motor improvements as assessed by the Unified Parkinson's Disease Rating Scale (UPDRS) motor score. Similarly, Feigin and colleagues [194] and Asanuma and colleagues [195] revealed at rest, a decrease in activation of the globus pallidus and subthalamic nuclei and an increase in cortical motor and premotor activity with administration of L-dopa, constituting a correction in the Parkinson's disease related motor pattern.

Investigating the effect of dopamine replacement on neuroimaging patterns in $\mathrm{PD}$ patients performing cognitive tasks, Cools and colleagues [196] found that Ldopa effectively normalized cerebral blood flow in PD patients compared to controls, decreasing activity in the right dorsolateral prefrontal cortex during performance of both planning and spatial working memory tasks compared with a visuomotor control task, and increasing cerebral blood flow in the right occipital lobe during a memory task relative to a control task. Mattay and colleagues [149] found that dopamine replacement increased activity in motor brain regions and decreased activity in the prefrontal cortical regions, constituting a correction of the PD pattern and correlating with decreased error rates on a working memory test. Fera and colleagues [197] showed that PD patients off medication had increased Stroop interferencerelated activity in anterior cingulate and presupplementary motor cortex compared to controls. L-dopa administration attenuated responses in these regions and increased activity 
in prefrontal cortex, which correlated with more accurate Stroop performance. Jahanshahi and colleagues [158] found that in PD patients off medication, motor timing tasks activated bilateral cerebellum, right thalamus, and left midbrain relative to a control, reaction time task whereas for healthy volunteers this contrast revealed significantly increased activity in left medial prefrontal cortex, right hippocampus, bilateral angular gyrus, left posterior cingulate, and left nucleus accumbens and caudate. Administration of a dopamine agonist increased activity in prefrontal regions in PD patients and was associated with improved performance. In PD patients, administration of apomorphine during performance of the TOL revealed greater deactivation of ventro-medial prefrontal cortex, a region belonging to the default network, as a function of task complexity [160]. Finally, Jubault and colleagues [198] found that treatment with dopaminergic therapy had no effect on brain activity in regions implicated in planning a set shift (i.e., caudate nucleus, ventrolateral, posterior, and dorsolaterlal prefrontal cortex) in PD patients but increased activity in the premotor cortex, essentially normalizing the pattern observed for setshift execution.

\subsection{Impairment of Neuroimaging Patterns with Dopaminergic} Therapy in PD Patients. In some cases, administration of L-dopa is associated with abnormal patterns of brain activity in PD. Feigin and colleagues [162] found that administration of L-dopa reduced sequence learning and was associated with enhanced activation in the right premotor and decreased activity in the ipsilateral occipital association area compared to controls. Argyelan and colleagues [199] showed that L-dopa diminished learning-related ventromedial prefrontal cortex suppression in a sequence learning task compared to unmedicated PD patients and healthy controls. In PD patients, administration of L-dopa correlated with greater attenuation of the dorsal striatum, insula, subgenual cingulate, and lateral orbitofrontal cortices for delayed relative to more immediate rewards, paralleling the behavioural result of increased impulsivity and temporal discounting relative to placebo [90]. Steeves and colleagues [200] found decreased binding of the D2 receptor ligand RAC in the ventral striatum in PD patients with pathological gambling relative to $\mathrm{PD}$ patients not known for pathological gambling, following administration of a dopamine agonist during gambling and control tasks. Along similar lines, using $\mathrm{H}_{2}\left[{ }^{15} \mathrm{O}\right]$ PET to measure regional cerebral blood flow as an index of regional brain activity during decision making with probabilistic feedback, van Eimeren and colleagues [201] compared PD patients with and without DA-induced pathological gambling before and after apomorphine administration. Pathological gamblers evidenced a dopamine agonist-induced attenuation of impulse control and response inhibition brain regions such as lateral orbitofrontal cortex, rostral cingulate, amygdala, and external pallidum whereas nongamblers revealed increased activity in these brain regions with administration of a dopaminergic agonist. These results suggest good correlation between the general behavioural effects and changes in neural activity precipitated by dopamine replacement, but highlight that individual differences can also augment or mitigate these correlations. Finally, Delaveau and colleagues [202] investigated the effect of L-dopa on brain regions associated with facial emotion recognition. They found that L-dopa decreased task-associated amygdala activation in PD patients.

5.3. Effect of Dopamine Modulation in Healthy Controls. In healthy elderly volunteers, administration of apomorphine, a dopamine agonist, resulted in improved performance on the TOL task [160]. Performance on TOL produced deactivation in ventro-medial prefrontal cortex and posterior cingulate cortex, regions belonging to the default mode network, both on and off medication. On apomorphine, there was an inverse correlation between task complexity and ventromedial prefrontal cortex [160]. In this example, a dopamine agonist improved performance and enhanced connectivity of underlying brain networks. Given that these patients were elderly, the authors speculated that as aging is related to dopamine cell loss, apomorphine could have corrected a clinically nonmanifest dopaminergic deficit in their controls.

In most cases, however, dopamine modulation in healthy controls produces impairments in patterns of brain activity. L-dopa administration increased functional connectivity among the putamen, cerebellum, and brainstem, and between the ventral striatum and ventrolateral prefrontal cortex activity. It disrupted ventral striatum and dorsal caudate functional connectivity with the default mode network, however [203]. Delaveau and colleagues [204, 205] showed that L-dopa administration reduced bilateral amygdala activity, a region reciprocally connected to ventral striatum, in healthy elderly volunteers performing a facial emotional recognition task. Finally, Nagano-Saito and colleagues [181] found changes in brain activity, which correlated with performance of the WCST, in healthy controls after consuming a drink deficient in the dopamine precursors tyrosine and phenylalanine compared to after they consumed a drink balanced in amino acids. Following the balanced drink, greater connectivity occurred between the frontal lobes and striatum, correlating with faster set-shift response times, and deactivation was noted in areas normally suppressed during attention-demanding tasks, including the medial prefrontal cortex, posterior cingulate cortex, and hippocampus. Following the dopamine precursor-depleted drink, fronto-striatal connectivity was abolished and deactivations in medial prefrontal cortex, posterior cingulate, and hippocampus were no longer observed, associated with longer set shifting response times.

5.4. Summary: Effect of L-Dopa Administration on Neuroimaging Results. Overall, these results are consistent with the notion that dopamine replacement normalizes activity in the dorsal striatum and cortico-striatal networks that implicate dorsal striatum, both at rest and during performance of cognitive tasks. These changes consist of increases in some cortical regions and decreases in others, correlating with improved performance on a variety of cognitive tasks such as spatial working memory, selective attention, planning, and set shifting. 
Dysfunctional patterns of brain activity precipitated by dopamine replacement in PD are noted exclusively for ventral striatum-mediated processes. Although only Steeves and colleagues [200] observed direct dopamine enhancement in the ventral striatum with dopamine agonist administration, abnormal patterns of activity produced by dopaminergic medication in PD patients solely implicated brain regions that are reciprocally connected to the ventral striatum. That is, reduced learning-related suppression in ventromedial prefrontal cortex occurred during sequence learning, increased activation of amygdala was noted on tests of facial emotional recognition, and greater attenuation of impulse control and response inhibition regions such as the dorsal striatum, insula, cingulate, and orbitofrontal cortex, were observed during more impulsive decisions in PD patients treated with dopaminergic medications. The studies reviewed here replicate the behavioural studies of dopamine replacement in cognition and confirm that those cognitive functions impaired by dopaminergic therapy in PD are related to changes in the ventral striatum and cortical networks that implicate the ventral striatum. The effect of dopamine supplementation in healthy controls on neural activity in the ventral striatum-associated cortical networks exactly mirrors the changes noted in PD, in line with the ventral striatum dopamine over-supply account of cognitive functions that worsen with treatment in PD.

We found rare direct but significant indirect evidence that dopamine replacement improves some aspects of cognition by remediating dorsal striatum function and worsens others by inducing pathological activity in ventral striatum. Studies are needed that directly contrast dorsal versus ventral striatum-mediated cognitive functions and associated neural activity in the same PD patients, on and off medication. Contrasting changes in brain activity noted for patients early in the disease course relative to those observed with more advanced disease will also enhance our understanding of how the relation between dopamine replacement and these divergent cognitive functions evolve in PD.

\section{General Discussion}

Cognitive dysfunction has long been recognized as a feature of PD. Cognitive functions are increasingly attributed to the basal ganglia [12-17, 19]. Dopamine replacement therapy has contrasting effects on different cognitive functions. In the current review, we present evidence that improvements with dopamine replacement arise for cognitive processes that are mediated by the dopamine-depleted dorsal striatum. In contrast, cognitive operations that are impaired by dopaminergic therapy are supported by the relatively spared ventral striatum.

Selecting among alternative stimuli and responses, particularly when there is high conflict or when enacting a decision requires disregarding previously relevant stimulus dimensions or responses, is improved by dopamine replacement. Dopaminergic therapy also remediates longterm memory retrieval, planning, visuo-spatial processing, as well as time estimation and motor-timing deficits. These cognitive functions are ascribed to the dorsal striatum in studies of patients with dorsal striatum lesions and in investigations of healthy controls using functional neuroimaging. Neuroimaging studies in PD confirm the notion that dopamine replacement improves cognitive functions mediated by dorsal striatum. Dopamine replacement normalizes activity in the dorsal striatum as well as in cortical networks involving dorsal striatum both at rest and during performance of cognitive tasks. These changes in neural activity are associated with improvements in cognitive performance.

In contrast, numerous studies reveal impaired probabilistic, associative, and sequence learning, decreased attentional orienting, as well as poorer facial emotional recognition in PD patients on relative to off dopamine replacement therapy. Impulsivity is enhanced to a pathological degree in PD patients on dopaminergic therapy. Studies of patients with ventral striatum lesions and neuroimaging investigations in healthy volunteers demonstrate that these behavioural phenomena are mediated by the ventral striatum. Imaging studies in PD on and off medication are therefore also consistent with the framework presented here for understanding medication effects on cognition in PD. Neuroimaging studies in PD patients in the off state confirm that ventral striatum and its cortical networks are unperturbed. Administration of dopaminergic therapy produces abnormal patterns of brain activity, with an increase in ventral striatal dopamine having been noted and alterations in levels of activation of cortical regions that are reciprocally connected to the ventral striatum being frequently observed. These neuroimaging changes are associated with behavioural impairments in ventral striatum-mediated cognitive processes. In line with claims that the ventral striatum receives adequate dopamine innervation early in PD and that dopamine supplementation oversupplies this region resulting in abnormal ventral-striatum mediated behaviour, the neuroimaging and behavioural consequences of dopamine supplementation in healthy controls with respect to learning, facial emotion recognition, and impulse control, exactly mirror those obtained with PD patients.

Although ventral-striatum mediated cognitive processes and their neural correlates are consistently adversely affected by dopamine supplementation in $\mathrm{PD}$, medication-induced effects in learning, orienting, and facial emotional recognition suggest reduced, whereas increased impulsivity reflects enhanced ventral striatum function. We speculate that these contrasting effects of dopamine replacement on ventral striatum-mediated cognitive functions relate to their differential reliance on graded versus absolute ventral striatum dopamine levels. Whereas dopamine replacement resulting in excessive dopamine concentration in ventral striatum conceivably disrupts processes that are informed by subtle relative or phasic changes in dopamine level-perhaps learning, orienting, and emotion discrimination, it might pathologically enhance processes that are governed by absolute or tonic dopamine signals. We submit that rapid decision making, guided by heuristics rather than complete consideration of all determinants and consequences of behaviour (i.e., impulsivity) is enhanced to a detrimental extent by dopamine replacement in PD. 
The cognitive profile in PD has many determinants. The importance of each of these factors evolves over the disease course. Some cognitive deficits owe to dopamine deficiency in the dorsal striatum, which are at least partially remediated by optimal dopaminergic therapy. In addition, dopamine overdose of the ventral striatum reduces some functions and heightens others to a pathological degree in PD patients receiving dopamine replacement. As functional neuroimaging studies demonstrate, dopamine deficiencies and excesses in the dorsal and ventral striatum, as a function of medication status, correlate with aberrant patterns of neural activity in cortical networks that are directly or even indirectly regulated by these respective brain regions. Although not addressed in this paper, cognitive dysfunction in PD also results from degeneration of cortex and other neurotransmitter systems, especially with advancing disease. Further, cortical regions receiving dopamine input from VTA, such as prefrontal and limbic regions, are also likely overdosed to varying extents by dopamine replacement in PD, impacting cognitive functions that they mediate. Finally, dopamine agonists and L-dopa have distinct mechanisms of action with somewhat different consequences on cognition [206], an issue glossed over in this paper. In light of numerous variables interacting to produce patterns of cognitive dysfunction and sparing in PD, given that these variables are differentially affected by dopaminergic therapy in general and by type of therapy specifically, and finally, because these interactions evolve over disease course, the framework adopted in this paper is clearly an over-simplification. That notwithstanding, it accommodates and explains an impressive array of cognitive and neuroimaging findings, providing a basic tenet for predicting and understanding the effect of dopamine replacement therapy on cognition in PD.

\subsection{Controversies and Areas Warranting Further Investigation.} Our review brings to light a number of inconsistencies as well as areas that warrant further consideration. Although dorsal striatum is implicated in selective attention and decision making, the specific aspect of these situations that depends upon the dorsal striatum remains somewhat unclear. Occasionally these executive functions are unimpaired in patients with dorsal striatum lesion or PD, and are not associated with preferential activation of the dorsal striatum using neuroimaging. We submit that decisions requiring integration of multiple dimensions, particularly those that require resolving conflicting influences on responding, depend to the greatest extent on the dorsal striatum. We predict that these instances will be most improved by dopamine replacement. Further investigation, however, is required.

With respect to the ventral striatum, whether this region mediates encoding for implicit or explicit uses of memory differentially has not yet been directly investigated although our survey of the literature does not suggest such specificity. Further, although the effects of dopamine replacement are consistently adverse with respect to ventral-striatum mediated behavior, some functions are reduced whereas others are pathologically enhanced. We argue that this relates to whether a function derives from graded, phasic dopamine responses, which bolus dopamine treatment will interrupt, versus absolute dopaminergic tone that will be heightened by dopamine replacement. Direct empirical investigations of this hypothesis are needed.

Finally, studies aimed specifically at contrasting dorsal versus ventral striatum-mediated cognitive functions in $\mathrm{PD}$ patients on and off medication relative to controls using neuroimaging are lacking. These studies will provide a greater understanding of the changes within the dorsal and ventral striato-cortical networks that occur in $\mathrm{PD}$ and how these are modulated by dopamine therapy. Investigations of how these interactions evolve over the disease course will also improve our understanding of the effect of dopamine replacement on cognition in PD.

\section{Conclusion}

This review highlights the fact that currently, titration of therapy in PD is geared to optimizing dorsal striatummediated motor symptoms, at the expense of ventral striatum-mediated operations. This consequence is only beginning to be recognized and the impact fully appreciated. Enhanced awareness of the differential effects of dopamine replacement on disparate cognitive functions will translate to medication strategies that take into account both those symptoms that dopamine replacement might improve versus hinder. Ultimately, this knowledge will lead clinicians to survey a broader range of symptoms and signs in determining optimal therapy based on individual patient priorities.

\section{References}

[1] R. G. Brown and C. D. Marsden, "How common is dementia in Parkinson's disease?" The Lancet, vol. 2, no. 8414, pp. 1262-1265, 1984.

[2] D. Aarsland, J. Zaccai, and C. Brayne, "A systematic review of prevalence studies of dementia in Parkinson's disease," Movement Disorders, vol. 20, no. 10, pp. 1255-1263, 2005.

[3] L. M. L. de Lau, C. M. A. Schipper, A. Hofman, P. J. Koudstaal, and M. M. B. Breteler, "Prognosis of Parkinson disease. Risk of dementia and mortality: the rotterdam study," Archives of Neurology, vol. 62, no. 8, pp. 1265-1269, 2005.

[4] R. J. Athey, R. W. Porter, and R. W. Walker, "Cognitive assessment of a representative community population with Parkinson's disease (PD) using the Cambridge Cognitive Assessment-Revised (CAMCOG-R)," Age and Ageing, vol. 34, no. 3, pp. 268-273, 2005.

[5] P. Hobson and J. Meara, "Risk and incidence of dementia in a cohort of older subjects with Parkinson's disease in the United Kingdom," Movement Disorders, vol. 19, no. 9, pp. 1043-1049, 2004.

[6] D. Aarsland and M. W. Kurz, "The epidemiology of dementia associated with Parkinson's disease," Brain Pathology, vol. 20, no. 3, pp. 633-639, 2010.

[7] L. Parkkinen, T. Kauppinen, T. Pirttilä, J. M. Autere, and I. Alafuzoff, " $\alpha$-synuclein pathology does not predict extrapyramidal symptoms or dementia," Annals of Neurology, vol. 57, no. 1, pp. 82-91, 2005.

[8] L. Parkkinen, T. Pirttilâ, and I. Alafuzoff, "Applicability of current staging/categorization of $\alpha$-synuclein pathology and their clinical relevance," Acta Neuropathologica, vol. 115, no. 4, pp. 399-407, 2008. 
[9] D. Weisman, M. Cho, C. Taylor, A. Adame, L. J. Thal, and L. A. Hansen, "In dementia with Lewy bodies, Braak stage determines phenotype, not Lewy body distribution," Neurology, vol. 69, no. 4, pp. 356-359, 2007.

[10] K. A. Jellinger, "A critical reappraisal of current staging of Lewy-related pathology in human brain," Acta Neuropathologica, vol. 116, no. 1, pp. 1-16, 2008.

[11] K. A. Jellinger, "A critical evaluation of current staging of $\alpha$ synuclein pathology in Lewy body disorders," Biochimica et Biophysica Acta, vol. 1792, no. 7, pp. 730-740, 2009.

[12] N. Ali, D. W. Green, F. Kherif, J. T. Devlin, and C. J. Price, "The role of the left head of caudate in suppressing irrelevant words," Journal of Cognitive Neuroscience, vol. 22, no. 10, pp. 2369-2386, 2010.

[13] R. Cools, "Dopaminergic modulation of cognitive functionimplications for L-DOPA treatment in Parkinson's disease," Neuroscience and Biobehavioral Reviews, vol. 30, no. 1, pp. 123, 2006.

[14] O. Monchi, M. Petrides, J. Doyon, R. B. Postuma, K. Worsley, and A. Dagher, "Neural bases of set-shifting deficits in Parkinson's disease," Journal of Neuroscience, vol. 24, no. 3, pp. 702-710, 2004.

[15] O. Monchi, M. Petrides, A. P. Strafella, K. J. Worsley, and J. Doyon, "Functional role of the basal ganglia in the planning and execution of actions," Annals of Neurology, vol. 59, no. 2, pp. 257-264, 2006.

[16] J.-S. Provost, M. Petrides, and O. Monchi, "Dissociating the role of the caudate nucleus and dorsolateral prefrontal cortex in the monitoring of events within human working memory," European Journal of Neuroscience, vol. 32, no. 5, pp. 873-880, 2010.

[17] C. A. Seger, E. J. Peterson, C. M. Cincotta, D. Lopez-Paniagua, and C. W. Anderson, "Dissociating the contributions of independent corticostriatal systems to visual categorization learning through the use of reinforcement learning modeling and Granger causality modeling," NeuroImage, vol. 50, no. 2, pp. 644-656, 2010.

[18] C. H. Adler, D. J. Connor, J. G. Hentz et al., "Incidental lewy body disease: clinical comparison to a control cohort," Movement Disorders, vol. 25, no. 5, pp. 642-646, 2010.

[19] K. A. Jellinger, "Neuropathology in Parkinson's disease with mild cognitive impairment," Acta Neuropathologica, vol. 120, no. 6, pp. 829-830, 2010.

[20] M. D. Humphries and T. J. Prescott, "The ventral basal ganglia, a selection mechanism at the crossroads of space, strategy, and reward," Progress in Neurobiology, vol. 90, no. 4, pp. 385-417, 2010.

[21] P. Voorn, L. J. M. J. Vanderschuren, H. J. Groenewegen, T. W. Robbins, and C. M. A. Pennartz, "Putting a spin on the dorsal-ventral divide of the striatum," Trends in Neurosciences, vol. 27, no. 8, pp. 468-474, 2004.

[22] J. R. Wickens, C. S. Budd, B. I. Hyland, and G. W. Arbuthnott, "Striatal contributions to reward and decision making: making sense of regional variations in a reiterated processing matrix," Annals of the New York Academy of Sciences, vol. 1104, pp. 192-212, 2007.

[23] R. Djaldetti, M. Lorberboym, Y. Karmon, T. A. Treves, I. Ziv, and E. Melamed, "Residual striatal dopaminergic nerve terminals in very long-standing Parkinson's disease: a single photon emission computed tomography imaging study," Movement Disorders. In press.

[24] J. M. Fearnley and A. J. Lees, "Ageing and Parkinson's disease: substantia nigra regional selectivity," Brain, vol. 114, no. 5, pp. 2283-2301, 1991.
[25] S. J. Kish, K. Shannak, and O. Hornykiewicz, "Uneven pattern of dopamine loss in the striatum of patients with idiopathic Parkinson's disease. Pathophysiologic and clinical implications," The New England Journal of Medicine, vol. 318, no. 14 , pp. 876-880, 1988.

[26] D. A. McRitchie, H. R. Cartwright, and G. M. Halliday, "Specific A10 dopaminergic nuclei in the midbrain degenerate in Parkinson's disease," Experimental Neurology, vol. 144, no. 1, pp. 202-213, 1997.

[27] R. Cools, R. A. Barker, B. J. Sahakian, and T. W. Robbins, "Enhanced or impaired cognitive function in Parkinson's disease as a function of dopaminergic medication and task demands," Cerebral Cortex, vol. 11, no. 12, pp. 1136-1143, 2001.

[28] A. M. Gotham, R. G. Brown, and C. D. Marsden, "Levodopa treatment may benefit or impair 'frontal' function in Parkinson's disease," The Lancet, vol. 2, no. 8513, pp. 970-971, 1986.

[29] A. M. Gotham, R. G. Brown, and C. D. Marsden, "'Frontal' cognitive function in patients with Parkinson's disease 'on' and 'off' levodopa," Brain, vol. 111, no. 2, pp. 299-321, 1988.

[30] M. A. Mehta, F. F. Manes, G. Magnolfi, B. J. Sahakian, and T. W. Robbins, "Impaired set-shifting and dissociable effects on tests of spatial working memory following the dopamine D receptor antagonist sulpiride in human volunteers," Psychopharmacology, vol. 176, no. 3-4, pp. 331-342, 2004.

[31] M. A. Mehta, R. Swainson, A. D. Ogilvie, B. Sahakian, and T. W. Robbins, "Improved short-term spatial memory but impaired reversal learning following the dopamine $\mathrm{D}_{2}$ agonist bromocriptine in human volunteers," Psychopharmacology, vol. 159, no. 1, pp. 10-20, 2002.

[32] R. Swainson, R. D. Rogers, B. J. Sahakian, B. A. Summers, C. E. Polkey, and T. W. Robbins, "Probabilistic learning and reversal deficits in patients with Parkinson's disease or frontal or temporal lobe lesions: possible adverse effects of dopaminergic medication," Neuropsychologia, vol. 38, no. 5, pp. 596-612, 2000.

[33] T. Zhang, L. Zhang, Y. Liang, A. G. Siapas, F. M. Zhou, and J. A. Dani, "Dopamine signaling differences in the nucleus accumbens and dorsal striatum exploited by nicotine," Journal of Neuroscience, vol. 29, no. 13, pp. 4035-4043, 2009.

[34] S. E. Leh, M. M. Chakravarty, and A. Ptito, "The connectivity of the human pulvinar: a diffusion tensor imaging tractography study," International Journal of Biomedical Imaging, vol. 2008, no. 1, Article ID 789539, 2008.

[35] R. L. Cowan and C. J. Wilson, "Spontaneous firing patterns and axonal projections of single corticostriatal neurons in the rat medial agranular cortex," Journal of Neurophysiology, vol. 71, no. 1, pp. 17-32, 1994.

[36] J. C. Houk, "Agents of the mind," Biological Cybernetics, vol. 92, no. 6, pp. 427-437, 2005.

[37] A. E. Kincaid, T. Zheng, and C. J. Wilson, "Connectivity and convergence of single corticostriatal axons," Journal of Neuroscience, vol. 18, no. 12, pp. 4722-4731, 1998.

[38] T. Zheng and C. J. Wilson, "Corticostriatal combinatorics: the implications of corticostriatal axonal arborizations," Journal of Neurophysiology, vol. 87, no. 2, pp. 1007-1017, 2002.

[39] T. Benke, M. Delazer, L. Bartha, and A. Auer, "Basal ganglia lesions and the theory of fronto-subcortical loops: neuropsychological findings in two patients with left caudate lesions," Neurocase, vol. 9, no. 1, pp. 70-85, 2003. 
[40] R. Cools, R. B. Ivry, and M. D’Esposito, “The human striatum is necessary for responding to changes in stimulus relevance," Journal of Cognitive Neuroscience, vol. 18, no. 12, pp. 19731983, 2006.

[41] S. W. Ell, N. L. Marchant, and R. B. Ivry, "Focal putamen lesions impair learning in rule-based, but not informationintegration categorization tasks," Neuropsychologia, vol. 44, no. 10, pp. 1737-1751, 2006.

[42] E. Yehene, N. Meiran, and N. Soroker, "Basal ganglia play a unique role in task switching within the frontal-subcortical circuits: evidence from patients with focal lesions," Journal of Cognitive Neuroscience, vol. 20, no. 6, pp. 1079-1093, 2008.

[43] S. Aglioti, A. Beltramello, F. Girardi, and F. Fabbro, "Neurolinguistic and follow-up study of an unusual pattern of recovery from bilingual subcortical aphasia," Brain, vol. 119, no. 5, pp. 1551-1564, 1996.

[44] M. Rieger, S. Gauggel, and K. Burmeister, "Inhibition of ongoing responses following frontal, nonfrontal, and basal ganglia lesions," Neuropsychology, vol. 17, no. 2, pp. 272-282, 2003.

[45] P. Thoma, B. Koch, K. Heyder, M. Schwarz, and I. Daum, "Subcortical contributions to multitasking and response inhibition," Behavioural Brain Research, vol. 194, no. 2, pp. 214-222, 2008.

[46] A. K. Troyer, S. E. Black, M. L. Armilio, and M. Moscovitch, "Cognitive and motor functioning in a patient with selective infarction of the left basal ganglia: evidence for decreased non-routine response selection and performance," Neuropsychologia, vol. 42, no. 7, pp. 902-911, 2004.

[47] M. Ullsperger and D. Y. von Cramon, "The role of intact frontostriatal circuits in error processing," Journal of Cognitive Neuroscience, vol. 18, no. 4, pp. 651-664, 2006.

[48] C. Bellebaum, B. Koch, M. Schwarz, and I. Daum, "Focal basal ganglia lesions are associated with impairments in reward-based reversal learning," Brain, vol. 131, no. 3, pp. 829-841, 2008.

[49] E. Vakil, H. Blachstein, and N. Soroker, "Differential effect of right and left basal ganglionic infarctions on procedural learning," Cognitive and Behavioral Neurology, vol. 17, no. 2, pp. 62-73, 2004.

[50] J. H. Ricker and S. R. Millis, "Differential visuospatial dysfunction following striatal, frontal white matter, or posterior thalamic infarction," International Journal of Neuroscience, vol. 84, no. 1-4, pp. 75-85, 1996.

[51] K. Schmidtke, H. Manner, R. Kaufmann, and H. Schmolck, "Cognitive procedural learning in patients with frontostriatal lesions," Learning and Memory, vol. 9, no. 6, pp. 419429, 2002.

[52] C. Y. Su, H. M. Chen, A. L. Kwan, Y. H. Lin, and N. W. Guo, "Neuropsychological impairment after hemorrhagic stroke in basal ganglia," Archives of Clinical Neuropsychology, vol. 22, no. 4, pp. 465-474, 2007.

[53] E. Vakil, S. Kahan, M. Huberman, and A. Osimani, "Motor and non-motor sequence learning in patients with basal ganglia lesions: the case of serial reaction time (SRT)," Neuropsychologia, vol. 38, no. 1, pp. 1-10, 2000.

[54] J. F. Hay, M. Moscovitch, and B. Levine, "Dissociating habit and recollection: evidence from Parkinson's disease, amnesia and focal lesion patients," Neuropsychologia, vol. 40, no. 8, pp. 1324-1334, 2002.

[55] D. Copland, "The basal ganglia and semantic engagement: potential insights from semantic priming in individuals with subcortical vascular lesions, Parkinson's disease, and cortical lesions," Journal of the International Neuropsychological Society, vol. 9, no. 7, pp. 1041-1052, 2003.

[56] O. Godefroy, M. Rousseaux, J. P. Pruvo, M. Cabaret, and D. Leys, "Neuropsychological changes related to unilateral lenticulostriate infarcts," Journal of Neurology Neurosurgery and Psychiatry, vol. 57, no. 4, pp. 480-485, 1994.

[57] F. Fabbro, A. Clarici, and A. Bava, "Effects of left basal ganglia lesions on language production," Perceptual and Motor Skills, vol. 84, no. 3, part 2, pp. 1291-1298, 1996.

[58] D. E. Jacome, "Case report: subcortical prosopagnosia and anosognosia," American Journal of the Medical Sciences, vol. 292, no. 6, pp. 386-388, 1986.

[59] L. A. Boyd and C. J. Winstein, "Providing explicit information disrupts implicit motor learning after basal ganglia stroke," Learning and Memory, vol. 11, no. 4, pp. 388-396, 2004.

[60] C. Exner, J. Koschack, and E. Irle, "The differential role of premotor frontal cortex and basal ganglia in motor sequence learning: evidence from focal basal ganglia lesions," Learning and Memory, vol. 9, no. 6, pp. 376-386, 2002.

[61] J. C. Shin, P. Aparicio, and R. B. Ivry, "Multidimensional sequence learning in patients with focal basal ganglia lesions," Brain and Cognition, vol. 58, no. 1, pp. 75-83, 2005.

[62] S. Kéri, S. Beniczky, E. Vörös, Z. Janka, G. Benedek, and L. Vécsei, "Dissociation between attentional set shifting and habit learning: a longitudinal case study," Neurocase, vol. 8, no. 3, pp. 219-225, 2002.

[63] J. Grinband, J. Hirsch, and V. P. Ferrera, "A neural representation of categorization uncertainty in the human brain," Neuron, vol. 49, no. 5, pp. 757-763, 2006.

[64] O. Monchi, M. Petrides, V. Petre, K. Worsley, and A. Dagher, "Wisconsin card sorting revisited: distinct neural circuits participating in different stages of the task identified by event-related functional magnetic resonance imaging," Journal of Neuroscience, vol. 21, no. 19, pp. 7733-7741, 2001.

[65] R. D. Rogers, T. C. Andrews, P. M. Grasby, D. J. Brooks, and T. W. Robbins, "Contrasting cortical and subcortical activations produced by attentional-set shifting and reversal learning in humans," Journal of Cognitive Neuroscience, vol. 12, no. 1, pp. 142-162, 2000.

[66] M. van Schouwenburg, E. Aarts, and R. Cools, "Dopaminergic modulation of cognitive control: distinct roles for the prefrontal cortex and the basal ganglia," Current Pharmaceutical Design, vol. 16, no. 18, pp. 2026-2032, 2010.

[67] H. Liu, Z. Hu, T. Guo, and D. Peng, "Speaking words in two languages with one brain: neural overlap and dissociation," Brain Research, vol. 1316, pp. 75-82, 2010.

[68] S. Vossel, R. Weidner, and G. R. Fink, "Dynamic coding of events within the inferior frontal gyrus in a probabilistic selective attention task," Journal of Cognitive Neuroscience, vol. 23, no. 2, pp. 414-424, 2011.

[69] J. P. Reiss, D. W. Campbell, W. D. Leslie et al., "The role of the striatum in implicit learning: a functional magnetic resonance imaging study," NeuroReport, vol. 16, no. 12, pp. 1291-1295, 2005.

[70] H. Kim, "Dissociating the roles of the default-mode, dorsal, and ventral networks in episodic memory retrieval," NeuroImage, vol. 50, no. 4, pp. 1648-1657, 2010.

[71] S. Helie, J. L. Roeder, and F. G. Ashby, "Evidence for cortical automaticity in rule-based categorization," Journal of Neuroscience, vol. 30, no. 42, pp. 14225-14234, 2010. 
[72] R. Daniel, G. Wagner, K. Koch, J. R. Reichenbach, H. Sauer, and R. G. Schlösser, "Assessing the neural basis of uncertainty in perceptual category learning through varying levels of distortion," Journal of Cognitive Neuroscience. In press.

[73] N. D. Daw and K. Doya, "The computational neurobiology of learning and reward," Current Opinion in Neurobiology, vol. 16, no. 2, pp. 199-204, 2006.

[74] M. R. Delgado, M. M. Miller, S. Inati, and E. A. Phelps, "An fMRI study of reward-related probability learning," NeuroImage, vol. 24, no. 3, pp. 862-873, 2005.

[75] H. Ohira, N. Ichikawa, M. Nomura et al., "Brain and autonomic association accompanying stochastic decisionmaking," NeuroImage, vol. 49, no. 1, pp. 1024-1037, 2010.

[76] J. T. Coull, R.-K. Cheng, and W. H. Meck, "Neuroanatomical and neurochemical substrates of timing," Neuropsychopharmacology, vol. 36, no. 1, pp. 3-25, 2011.

[77] JT. T. Coull and AC. C. Nobre, "Dissociating explicit timing from temporal expectation with fMRI," Current Opinion in Neurobiology, vol. 18, no. 2, pp. 137-144, 2008.

[78] A. M. Ferrandez, L. Hugueville, S. Lehéricy, J. B. Poline, C. Marsault, and V. Pouthas, "Basal ganglia and supplementary motor area subtend duration perception: an fMRI study," NeuroImage, vol. 19, no. 4, pp. 1532-1544, 2003.

[79] D. L. Harrington, L. A. Boyd, A. R. Mayer et al., "Neural representation of interval encoding and decision making," Cognitive Brain Research, vol. 21, no. 2, pp. 193-205, 2004.

[80] D. L. Harrington, J. L. Zimbelman, S. C. Hinton, and S. M. Rao, "Neural modulation of temporal encoding, maintenance, and decision processes," Cerebral Cortex, vol. 20, no. 6, pp. 1274-1285, 2010.

[81] M. Jahanshahi, C. R. G. Jones, G. Dirnberger, and C. D. Frith, "The substantia nigra pars compacta and temporal processing," Journal of Neuroscience, vol. 26, no. 47, pp. 12266-12273, 2006.

[82] I. Nenadic, C. Gaser, H. P. Volz, T. Rammsayer, F. Häger, and H. Sauer, "Processing of temporal information and the basal ganglia: new evidence from fMRI," Experimental Brain Research, vol. 148, no. 2, pp. 238-246, 2003.

[83] S. M. Rao, A. R. Mayer, and D. L. Harrington, "The evolution of brain activation during temporal processing," Nature Neuroscience, vol. 4, no. 3, pp. 317-323, 2001.

[84] S. M. Rao, "Distributed neural systems underlying the timing of movements," Journal of Neuroscience, vol. 17, no. 14, pp. 5528-5535, 1997.

[85] L. Y. L. Shih, W. J. Kuo, T. C. Yeh, O. J. L. Tzeng, and J. C. Hsieh, "Common neural mechanisms for explicit timing in the sub-second range," NeuroReport, vol. 20, no. 10, pp. 897901, 2009.

[86] T. Butler, J. Imperato-McGinley, H. Pan et al., "Sex differences in mental rotation: top-down versus bottom-up processing," NeuroImage, vol. 32, no. 1, pp. 445-456, 2006.

[87] J. B. Engelmann and D. Tamir, "Individual differences in risk preference predict neural responses during financial decision-making," Brain Research, vol. 1290, pp. 28-51, 2009.

[88] B. U. Forstmann, A. Anwander, A. Schäfer et al., "Corticostriatal connections predict control over speed and accuracy in perceptual decision making," Proceedings of the National Academy of Sciences of the United States of America, vol. 107, no. 36, pp. 15916-15920, 2010.

[89] A. Pine, B. Seymour, J. P. Roiser et al., "Encoding of marginal utility across time in the human brain," Journal of Neuroscience, vol. 29, no. 30, pp. 9575-9581, 2009.
[90] A. Pine, T. Shiner, B. Seymour, and R. J. Dolan, "Dopamine, time, and impulsivity in humans," Journal of Neuroscience, vol. 30, no. 26, pp. 8888-8896, 2010.

[91] G. Goldenberg, U. Schuri, O. Gromminger, and U. Arnold, "Basal forebrain amnesia: does the nucleus accumbens contribute to human memory?" Journal of Neurology Neurosurgery and Psychiatry, vol. 67, no. 2, pp. 163-168, 1999.

[92] A. J. Calder, J. Keane, A. D. Lawrence, and F. Manes, "Impaired recognition of anger following damage to the ventral striatum," Brain, vol. 127, no. 9, pp. 1958-1969, 2004.

[93] O. Martinaud, B. Perin, E. Gérardin et al., "Anatomy of executive deficit following ruptured anterior communicating artery aneurysm," European Journal of Neurology, vol. 16, no. 5, pp. 595-601, 2009.

[94] J. V. Filoteo, W. T. Maddox, A. N. Simmons et al., "Cortical and subcortical brain regions involved in rule-based category learning," NeuroReport, vol. 16, no. 2, pp. 111-115, 2005.

[95] C. A. Seger and C. M. Cincotta, "Dynamics of frontal, striatal, and hippocampal systems during rule learning," Cerebral Cortex, vol. 16, no. 11, pp. 1546-1555, 2006.

[96] M. Pessiglione, P. Petrovic, J. Daunizeau, S. Palminteri, R. J. Dolan, and C. D. Frith, "Subliminal instrumental conditioning demonstrated in the human brain," Neuron, vol. 59, no. 4, pp. 561-567, 2008.

[97] S. Bray and J. O’Doherty, "Neural coding of rewardprediction error signals during classical conditioning with attractive faces," Journal of Neurophysiology, vol. 97, no. 4, pp. 3036-3045, 2007.

[98] H. C. Breiter, I. Aharon, D. Kahneman, A. Dale, and P. Shizgal, "Functional imaging of neural responses to expectancy and experience of monetary gains and losses," Neuron, vol. 30, no. 2, pp. 619-639, 2001.

[99] P. F. Rodriguez, A. R. Aron, and R. A. Poldrack, "Ventralstriatal/nucleus-accumbens sensitivity to prediction errors during classification learning," Human Brain Mapping, vol. 27, no. 4, pp. 306-313, 2006.

[100] E. T. Rolls, C. McCabe, and J. Redoute, "Expected value, reward outcome, and temporal difference error representations in a probabilistic decision task," Cerebral Cortex, vol. 18, no. 3, pp. 652-663, 2008.

[101] M. Ullsperger and D. Y. von Cramon, "Error monitoring using external feedback: specific roles of the habenular complex, the reward system, and the cingulate motor area revealed by functional magnetic resonance imaging," Journal of Neuroscience, vol. 23, no. 10, pp. 4308-4314, 2003.

[102] A. N. Hampton and J. P. O’Doherty, “Decoding the neural substrates of reward-related decision making with functional MRI," Proceedings of the National Academy of Sciences of the United States of America, vol. 104, no. 4, pp. 1377-1382, 2007.

[103] C. Simões-Franklin, R. Hester, M. Shpaner, J. J. Foxe, and H. Garavan, "Executive function and error detection: the effect of motivation on cingulate and ventral striatum activity," Human Brain Mapping, vol. 31, no. 3, pp. 458-469, 2010.

[104] R. Cools, L. Clark, A. M. Owen, and T. W. Robbins, "Defining the neural mechanisms of probabilistic reversal learning using event-related functional magnetic resonance imaging," Journal of Neuroscience, vol. 22, no. 11, pp. 4563-4567, 2002.

[105] R. Cools, L. Clark, and T. W. Robbins, "Differential responses in human striatum and prefrontal cortex to changes in object and rule relevance," Journal of Neuroscience, vol. 24, no. 5, pp. 1129-1135, 2004. 
[106] C. M. Dodds, U. Müller, L. Clark, A. van Loon, R. Cools, and T. W. Robbins, "Methylphenidate has differential effects on blood oxygenation level-dependent signal related to cognitive subprocesses of reversal learning," Journal of Neuroscience, vol. 28, no. 23, pp. 5976-5982, 2008.

[107] J. C. Cooper and B. Knutson, "Valence and salience contribute to nucleus accumbens activation," NeuroImage, vol. 39, no. 1, pp. 538-547, 2008.

[108] A. Litt, H. Plassmann, B. Shiv, and A. Rangel, "Dissociating valuation and saliency signals during decision-making," Cerebral Cortex, vol. 21, no. 1, pp. 95-102, 2011.

[109] C. F. Zink, G. Pagnoni, M. E. Martin, M. Dhamala, and G. S. Berns, "Human striatal response to salient nonrewarding stimuli," Journal of Neuroscience, vol. 23, no. 22, pp. 8092 8097, 2003.

[110] J. W. Kable and P. W. Glimcher, "An "as soon as possible" effect in human intertemporal decision making: behavioral evidence and neural mechanisms," Journal of Neurophysiology, vol. 103, no. 5, pp. 2513-2531, 2010.

[111] C. Prévost, M. Pessiglione, E. Météreau, M.-L. Cléry-Melin, and J.-C. Dreher, "Separate valuation subsystems for delay and effort decision costs," Journal of Neuroscience, vol. 30, no. 42, pp. 14080-14090, 2010.

[112] M. R. van Schouwenburg, H. E.M. den Ouden, and R. Cools, "The human basal ganglia modulate frontal-posterior connectivity during attention shifting," Journal of Neuroscience, vol. 30, no. 29, pp. 9910-9918, 2010.

[113] J. A. Clithero, D. V. Smith, R. M. Carter, and S. A. Huettel, "Within- and cross-participant classifiers reveal different neural coding of information," NeuroImage. In press.

[114] B. Knutson and J. C. Cooper, "Functional magnetic resonance imaging of reward prediction," Current Opinion in Neurology, vol. 18, no. 4, pp. 411-417, 2005.

[115] K. Preuschoff, P. Bossaerts, and S. R. Quartz, "Neural differentiation of expected reward and risk in human subcortical structures," Neuron, vol. 51, no. 3, pp. 381-390, 2006.

[116] P. N. Tobler, P. C. Fletcher, E. T. Bullmore, and W. Schultz, "Learning-related human brain activations reflecting individual finances," Neuron, vol. 54, no. 1, pp. 167-175, 2007.

[117] J. Yacubian, J. Gläscher, K. Schroeder, T. Sommer, D. F. Braus, and C. Büchel, "Dissociable systems for gain- and loss-related value predictions and errors of prediction in the human brain," Journal of Neuroscience, vol. 26, no. 37, pp. 9530-9537, 2006.

[118] M. Ernst, E. E. Nelson, E. B. McClure et al., "Choice selection and reward anticipation: an fMRI study," Neuropsychologia, vol. 42, no. 12, pp. 1585-1597, 2004.

[119] S. C. Matthews, A. N. Simmons, S. D. Lane, and M. P. Paulus, "Selective activation of the nucleus accumbens during risktaking decision making," NeuroReport, vol. 15, no. 13, pp. 2123-2127, 2004.

[120] K. Basar, T. Sesia, H. Groenewegen, H. W.M. Steinbusch, V. Visser-Vandewalle, and Y. Temel, "Nucleus accumbens and impulsivity," Progress in Neurobiology, vol. 92, no. 4, pp. 533$557,2010$.

[121] N. R. Horn, M. Dolan, R. Elliott, J. F. W. Deakin, and P. W. R. Woodruff, "Response inhibition and impulsivity: an fMRI study," Neuropsychologia, vol. 41, no. 14, pp. 19591966, 2003.

[122] C. M. Kuhnen and B. Knutson, "The neural basis of financial risk taking," Neuron, vol. 47, no. 5, pp. 763-770, 2005.

[123] B. J. Weber and S. A. Huettel, "The neural substrates of probabilistic and intertemporal decision making," Brain Research, vol. 1234, pp. 104-115, 2008.
[124] A. R. Hariri, S. M. Brown, D. E. Williamson, J. D. Flory, H. de Wit, and S. B. Manuck, "Preference for immediate over delayed rewards is associated with magnitude of ventral striatal activity," Journal of Neuroscience, vol. 26, no. 51, pp. 13213-13217, 2006.

[125] C. C. Luhmann, M. M. Chun, D. J. Yi, D. Lee, and X. J. Wang, "Neural dissociation of delay and uncertainty in intertemporal choice," Journal of Neuroscience, vol. 28, no. 53, pp. 14459-14466, 2008.

[126] S. M. McClure, D. I. Laibson, G. Loewenstein, and J. D. Cohen, "Separate neural systems value immediate and delayed monetary rewards," Science, vol. 306, no. 5695, pp. 503-507, 2004.

[127] E. K. Diekhof and O. Gruber, "When desire collides with reason: functional interactions between anteroventral prefrontal cortex and nucleus accumbens underlie the human ability to resist impulsive desires," Journal of Neuroscience, vol. 30, no. 4, pp. 1488-1493, 2010.

[128] C. S. Monk, R. G. Klein, E. H. Telzer et al., "Amygdala and nucleus accumbens activation to emotional facial expressions in children and adolescents at risk for major depression," American Journal of Psychiatry, vol. 165, no. 1, pp. 90-98, 2008.

[129] A. Mühlberger, M. J. Wieser, A. B. M. Gerdes et al., "Stop looking angry and smile, please: start and stop of the very same facial expression differentially activate threat- and reward-related brain networks," Social Cognitive \& Affective Neuroscience. In press.

[130] X. Liang, L. A. Zebrowitz, and Y. Zhang, "Neural activation in the "reward circuit" shows a nonlinear response to facial attractiveness," Social Neuroscience, vol. 5, no. 3, pp. 320-334, 2010.

[131] R. Cools, R. A. Barker, B. J. Sahakian, and T. W. Robbins, "Mechanisms of cognitive set flexibility in Parkinson's disease," Brain, vol. 124, no. 12, pp. 2503-2512, 2001.

[132] R. Cools, R. A. Barker, B. J. Sahakian, and T. W. Robbins, "L-Dopa medication remediates cognitive inflexibility, but increases impulsivity in patients with Parkinson's disease," Neuropsychologia, vol. 41, no. 11, pp. 1431-1441, 2003.

[133] A. E. Hayes, M. C. Davidson, S. W. Keele, and R. D. Rafal, "Toward a functional analysis of the basal ganglia," Journal of Cognitive Neuroscience, vol. 10, no. 2, pp. 178-198, 1998.

[134] S. K. Shook, E. A. Franz, C. I. Higginson, V. L. Wheelock, and K. A. Sigvardt, "Dopamine dependency of cognitive switching and response repetition effects in Parkinson's patients," Neuropsychologia, vol. 43, no. 14, pp. 1990-1999, 2005.

[135] M.-H. Sohn, S. Ursu, J. R. Anderson, V. A. Stenger, and C. S. Carter, "The role of prefrontal cortex and posterior parietal cortex in task switching," Proceedings of the National Academy of Sciences of the United States of America, vol. 97, no. 24, pp. 13448-13453, 2000.

[136] A. J. Hood, S. C. Amador, A. E. Cain et al., "Levodopa slows prosaccades and improves antisaccades: an eye movement study in Parkinson's disease," Journal of Neurology, Neurosurgery and Psychiatry, vol. 78, no. 6, pp. 565-570, 2007.

[137] P. A. MacDonald, A. A. MacDonald, K. N. Seergobin et al., "Differential cognitive effects of dopamine replacement on ventral and dorsal striatum in Parkinson's disease: convergence from fMRI," submitted to Brain.

[138] S. J. G. Lewis, A. Slabosz, T. W. Robbins, R. A. Barker, and A. M. Owen, "Dopaminergic basis for deficits in working memory but not attentional set-shifting in Parkinson's disease," Neuropsychologia, vol. 43, no. 6, pp. 823-832, 2005. 
[139] A. Slabosz, S. J. G. Lewis, K. Smigasiewicz, B. Szymura, R. A. Barker, and A. M. Owen, "The role of learned irrelevance in attentional set-shifting impairments in Parkinson's disease," Neuropsychology, vol. 20, no. 5, pp. 578-588, 2006.

[140] D. M. E. Torta, L. Castelli, M. Zibetti, L. Lopiano, and G. Geminiani, "On the role of dopamine replacement therapy in decision-making, working memory, and reward in Parkinson's disease: does the therapy-dose matter?" Brain and Cognition, vol. 71, no. 2, pp. 84-91, 2009.

[141] A. Costa, A. Peppe, G. Dell’Agnello et al., "Dopaminergic modulation of visual-spatial working memory in Parkinson's disease," Dementia and Geriatric Cognitive Disorders, vol. 15, no. 2, pp. 55-66, 2003.

[142] A. Costa, A. Peppe, G. Dell'Agnello, C. Caltagirone, and G. A. Carlesimo, "Dopamine and cognitive functioning in de novo subjects with Parkinson's disease: effects of pramipexole and pergolide on working memory," Neuropsychologia, vol. 47, no. 5, pp. 1374-1381, 2009.

[143] A. Costa, A. Peppe, L. Brusa, C. Caltagirone, I. Gatto, and G. A. Carlesimo, "Dopaminergic modulation of prospective memory in Parkinson's disease," Behavioural Neurology, vol. 19, no. 1-2, pp. 45-48, 2008.

[144] A. Costa, A. Peppe, L. Brusa, C. Caltagirone, I. Gatto, and G. A. Carlesimo, "Levodopa improves time-based prospective memory in Parkinson's disease," Journal of the International Neuropsychological Society, vol. 14, no. 4, pp. 601-610, 2008.

[145] B. Hanna-Pladdy and K. M. Heilman, "Dopaminergic modulation of the planning phase of skill acquisition in Parkinson's disease," Neurocase, vol. 16, no. 2, pp. 182-190, 2010.

[146] P.-L. Tremblay, M.-A. Bedard, D. Langlois, P. J. Blanchet, M. Lemay, and M. Parent, "Movement chunking during sequence learning is a dopamine-dependant process: a study conducted in Parkinson's disease," Experimental Brain Research, vol. 205, no. 3, pp. 375-385, 2010.

[147] D. Shohamy, C. E. Myers, S. Grossman, J. Sage, and M. A. Gluck, "The role of dopamine in cognitive sequence learning: evidence from Parkinson's disease," Behavioural Brain Research, vol. 156, no. 2, pp. 191-199, 2005.

[148] R. Beato, R. Levy, B. Pillon et al., "Working memory in Parkinson's disease patients: clinical features and response to levodopa," Arquivos de Neuro-Psiquiatria, vol. 66, no. 2 A, pp. 147-151, 2008.

[149] V. S. Mattay, A. Tessitore, J. H. Callicott et al., "Dopaminergic modulation of cortical function in patients with Parkinson's disease," Annals of Neurology, vol. 51, no. 2, pp. 156-164, 2002.

[150] H. Mollion, J. Ventre-Dominey, P. F. Dominey, and E. Broussolle, "Dissociable effects of dopaminergic therapy on spatial versus non-spatial working memory in Parkinson's disease," Neuropsychologia, vol. 41, no. 11, pp. 1442-1451, 2003.

[151] K. W. Lange, T. W. Robbins, C. D. Marsden, M. James, A. M. Owen, and G. M. Paul, “L-Dopa withdrawal in Parkinson's disease selectively impairs cognitive performance in tests sensitive to frontal lobe dysfunction," Psychopharmacology, vol. 107, no. 2-3, pp. 394-404, 1992.

[152] S. Righi, M. P. Viggiano, M. Paganini, S. Ramat, and P. Marini, "Recognition of category-related visual stimuli in Parkinson's disease: before and after pharmacological treatment," Neuropsychologia, vol. 45, no. 13, pp. 2931-2941, 2007.

[153] M. A. Pastor, J. Artieda, M. Jahanshahi, and J. A. Obeso, "Time estimation and reproduction is abnormal in Parkinson's disease," Brain, vol. 115, no. 1, pp. 211-225, 1992.
[154] D. J. O’Boyle, J. S. Freeman, and F. W. J. Cody, "The accuracy and precision of timing of self-paced, repetitive movements in subjects with Parkinson's disease," Brain, vol. 119, no. 1, pp. 51-70, 1996.

[155] C. R. G. Jones, T. J. L. Malone, G. Dirnberger, M. Edwards, and M. Jahanshahi, "Basal ganglia, dopamine and temporal processing: performance on three timing tasks on and off medication in Parkinson's disease," Brain and Cognition, vol. 68, no. 1, pp. 30-41, 2008.

[156] D. L. Harrington, K. Y. Haaland, and N. Hermanowicz, "Temporal processing in the basal ganglia," Neuropsychology, vol. 12, no. 1, pp. 3-12, 1998.

[157] C. L. Elsinger, S. M. Rao, J. L. Zimbelman, N. C. Reynolds, K. A. Blindauer, and R. G. Hoffmann, "Neural basis for impaired time reproduction in Parkinson's disease: an fMRI study," Journal of the International Neuropsychological Society, vol. 9, no. 7, pp. 1088-1098, 2003.

[158] M. Jahanshahi, L. Wilkinson, H. Gahir, A. Dharminda, and D. A. Lagnado, "Medication impairs probabilistic classification learning in Parkinson's disease," Neuropsychologia, vol. 48, no. 4, pp. 1096-1103, 2010.

[159] E. M. Fine, D. C. Delis, B. M. Paul, and J. V. Filoteo, "Reduced verbal fluency for proper names in nondemented patients with Parkinson's disease: a quantitative and qualitative analysis," Journal of Clinical and Experimental Neuropsychology, vol. 33, no. 2, pp. 226-233, 2011.

[160] A. Nagano-Saito, J. Liu, J. Doyon, and A. Dagher, "Dopamine modulates default mode network deactivation in elderly individuals during the Tower of London task," Neuroscience Letters, vol. 458, no. 1, pp. 1-5, 2009.

[161] D. Shohamy, C. E. Myers, K. D. Geghman, J. Sage, and M. A. Gluck, "L-dopa impairs learning, but spares generalization, in Parkinson's disease," Neuropsychologia, vol. 44, no. 5, pp. 774-784, 2006.

[162] A. Feigin, M. F. Ghilardi, M. Carbon et al., "Effects of levodopa on motor sequence learning in Parkinson's disease," Neurology, vol. 60, no. 11, pp. 1744-1749, 2003.

[163] M. Seo, M. Beigi, M. Jahanshahi, and B. B. Averbeck, "Effects of dopamine medication on sequence learning with stochastic feedback in Parkinson's disease," Frontiers in Human Neuroscience, vol. 4, 36 pages, 2010.

[164] M. F. Ghilardi, A. S. Feigin, F. Battaglia et al., "L-Dopa infusion does not improve explicit sequence learning in Parkinson's disease," Parkinsonism and Related Disorders, vol. 13, no. 3, pp. 146-151, 2007.

[165] R. Dias, T. W. Robbins, and A. C. Roberts, "Dissociation in prefrontal cortex of affective and attentional shifts," Nature, vol. 380, no. 6569, pp. 69-72, 1996.

[166] S. Graef, G. Biele, L. K. Krugel et al., "Differential influence of levodopa on reward-based learning in Parkinson's disease," Frontiers in Human Neuroscience, vol. 4, p. 169, 2010.

[167] R. Tomer, J. Aharon-Peretz, and Z. Tsitrinbaum, "Dopamine asymmetry interacts with medication to affect cognition in Parkinson's disease," Neuropsychologia, vol. 45, no. 2, pp. 357-367, 2007.

[168] M. J. Frank, L. C. Seeberger, and R. C. O’Reilly, "By carrot or by stick: cognitive reinforcement learning in Parkinsonism," Science, vol. 306, no. 5703, pp. 1940-1943, 2004.

[169] A. H. Evans, A. D. Lawrence, J. Potts et al., "Relationship between impulsive sensation seeking traits, smoking, alcohol and caffeine intake, and Parkinson's disease," Journal of Neurology, Neurosurgery and Psychiatry, vol. 77, no. 3, pp. 317-321, 2006. 
[170] N. Ray and A. P. Strafella, "Dopamine, reward, and frontostriatal circuitry in impulse control disorders in Parkinson's disease: insights from functional imaging," Clinical EEG and Neuroscience, vol. 41, no. 2, pp. 87-93, 2010.

[171] D. Weintraub, J. Koester, M. N. Potenza et al., "Impulse control disorders in Parkinson disease: a cross-sectional study of 3090 patients," Archives of Neurology, vol. 67, no. 5, pp. 589-595, 2010.

[172] S. Fenu, J. Wardas, and M. Morelli, "Impulse control disorders and dopamine dysregulation syndrome associated with dopamine agonist therapy in Parkinson's disease," Behavioural Pharmacology, vol. 20, no. 5-6, pp. 363-379, 2009.

[173] T. Müller, S. Benz, and C. Börnke, "Delay of simple reaction time after levodopa intake,” Clinical Neurophysiology, vol. 112, no. 11, pp. 2133-2137, 2001.

[174] T. Müller, S. Benz, and H. Przuntek, "Apomorphine delays simple reaction time in Parkinsonian patients," Parkinsonism and Related Disorders, vol. 8, no. 5, pp. 357-360, 2002.

[175] J. G. Smith, D. N. Harper, D. Gittings, and D. Abernethy, "The effect of Parkinson's disease on time estimation as a function of stimulus duration range and modality," Brain and Cognition, vol. 64, no. 2, pp. 130-143, 2007.

[176] C. Breitenstein, C. Korsukewitz, A. Flöel, T. Kretzschmar, K. Diederich, and S. Knecht, "Tonic dopaminergic stimulation impairs associative learning in healthy subjects," Neuropsychopharmacology, vol. 31, no. 11, pp. 2552-2564, 2006.

[177] D. A. Pizzagalli, A. E. Evins, E. C. Schetter et al., "Single dose of a dopamine agonist impairs reinforcement learning in humans: behavioral evidence from a laboratory-based measure of reward responsiveness," Psychopharmacology, vol. 196, no. 2, pp. 221-232, 2008.

[178] D. L. Santesso, A. E. Evins, M. J. Frank, E. C. Schetter, R. Bogdan, and D. A. Pizzagalli, "Single dose of a dopamine agonist impairs reinforcement learning in humans: evidence from event-related potentials and computational modeling of striatal-cortical function," Human Brain Mapping, vol. 30, no. 7, pp. 1963-1976, 2009.

[179] A. Schnider, A. Guggisberg, L. Nahum, D. Gabriel, and S. Morand, "Dopaminergic modulation of rapid reality adaptation in thinking," Neuroscience, vol. 167, no. 3, pp. 583-587, 2010.

[180] M. Luciana, P. F. Collins, and R. A. Depue, “Opposing roles for dopamine and serotonin in the modulation of human spatial working memory functions," Cerebral Cortex, vol. 8, no. 3, pp. 218-226, 1998.

[181] A. Nagano-Saito, M. Leyton, O. Monchi, Y. K. Goldberg, Y. He, and A. Dagher, "Dopamine depletion impairs frontostriatal functional connectivity during a set-shifting task," Journal of Neuroscience, vol. 28, no. 14, pp. 3697-3706, 2008.

[182] D. Y. Kimberg, M. D’Esposito, and M. J. Farah, “Effects of bromocriptine on human subjects depend on working memory capacity," NeuroReport, vol. 8, no. 16, pp. 35813585, 1997.

[183] U. Müller, D. Y. von Cramon, and S. Pollmann, "D1-versus D2-receptor modulation of visuospatial working memory in humans," Journal of Neuroscience, vol. 18, no. 7, pp. 27202728, 1998.

[184] D. Eidelberg, "Metabolic brain networks in neurodegenerative disorders: a functional imaging approach," Trends in Neurosciences, vol. 32, no. 10, pp. 548-557, 2009.
[185] T. van Eimeren, O. Monchi, B. Ballanger, and A. P. Strafella, "Dysfunction of the default mode network in Parkinson disease: a functional magnetic resonance imaging study," Archives of Neurology, vol. 66, no. 7, pp. 877-883, 2009.

[186] M. E. Raichle, A. M. MacLeod, A. Z. Snyder, W. J. Powers, D. A. Gusnard, and G. L. Shulman, "A default mode of brain function," Proceedings of the National Academy of Sciences of the United States of America, vol. 98, no. 2, pp. 676-682, 2001.

[187] M. E. Raichle and A. Z. Snyder, "A default mode of brain function: a brief history of an evolving idea," NeuroImage, vol. 37, no. 4, pp. 1083-1090, 2007.

[188] V. L. Cropley, M. Fujita, W. Bara-Jimenez et al., "Preand post-synaptic dopamine imaging and its relation with frontostriatal cognitive function in Parkinson disease: PET studies with $\left[{ }^{11} \mathrm{C}\right] \mathrm{NNC} 112$ and $\left[{ }^{18} \mathrm{~F}\right] \mathrm{FDOPA}, "$ Psychiatry Research, vol. 163, no. 2, pp. 171-182, 2008.

[189] P. Jokinen, A. Brück, S. Aalto, S. Forsback, R. Parkkola, and J. O. Rinne, "Impaired cognitive performance in Parkinson's disease is related to caudate dopaminergic hypofunction and hippocampal atrophy," Parkinsonism and Related Disorders, vol. 15, no. 2, pp. 88-93, 2009.

[190] N. Sawamoto, P. Piccini, G. Hotton, N. Pavese, K. Thielemans, and D. J. Brooks, "Cognitive deficits and striato-frontal dopamine release in Parkinson's disease," Brain, vol. 131, no. 5, pp. 1294-1302, 2008.

[191] T. Schonberg, J. P. O’Doherty, D. Joel, R. Inzelberg, Y. Segev, and N. D. Daw, "Selective impairment of prediction error signaling in human dorsolateral but not ventral striatum in Parkinson's disease patients: evidence from a model-based fMRI study," NeuroImage, vol. 49, no. 1, pp. 772-781, 2010.

[192] O. Monchi, M. Petrides, B. Mejia-Constain, and A. P. Strafella, "Cortical activity in Parkinson's disease during executive processing depends on striatal involvement," Brain, vol. 130, no. 1, pp. 233-244, 2007.

[193] T. Wu, L. Wang, Y. Chen, C. Zhao, K. Li, and P. Chan, "Changes of functional connectivity of the motor network in the resting state in Parkinson's disease," Neuroscience Letters, vol. 460, no. 1, pp. 6-10, 2009.

[194] A. Feigin, M. Fukuda, V. Dhawan et al., "Metabolic correlates of levodopa response in Parkinson's disease," Neurology, vol. 57, no. 11, pp. 2083-2088, 2001.

[195] K. Asanuma, C. Tang, Y. Ma et al., "Network modulation in the treatment of Parkinson's disease," Brain, vol. 129, no. 10, pp. 2667-2678, 2006.

[196] R. Cools, E. Stefanova, R. A. Barker, T. W. Robbins, and A. M. Owen, "Dopaminergic modulation of high-level cognition in Parkinson's disease: the role of the prefrontal cortex revealed by PET," Brain, vol. 125, no. 3, pp. 584-594, 2002.

[197] F. Fera, G. Nicoletti, A. Cerasa et al., "Dopaminergic modulation of cognitive interference after pharmacological washout in Parkinson's disease," Brain Research Bulletin, vol. 74, no. 1-3, pp. 75-83, 2007.

[198] T. Jubault, L. Monetta, A. P. Strafella, A. L. Lafontaine, and O. Monchi, "L-dopa medication in Parkinson's disease restores activity in the motor cortico-striatal loop but does not modify the cognitive network," PLoS One, vol. 4, no. 7, Article ID e6154, 2009.

[199] M. Argyelan, M. Carbon, M. F. Ghilardi et al., "Dopaminergic suppression of brain deactivation responses during sequence learning," Journal of Neuroscience, vol. 28, no. 42, pp. 10687-10695, 2008. 
[200] T. D. L. Steeves, J. Miyasaki, M. Zurowski et al., "Increased striatal dopamine release in Parkinsonian patients with pathological gambling: a $\left[{ }^{11} \mathrm{C}\right]$ raclopride PET study," Brain, vol. 132, no. 5, pp. 1376-1385, 2009.

[201] T. van Eimeren, G. Pellecchia, R. Cilia et al., "Drug-induced deactivation of inhibitory networks predicts pathological gambling in PD," Neurology, vol. 75, no. 19, pp. 1711-1716, 2010.

[202] P. Delaveau, P. Salgado-Pineda, T. Witjas et al., "Dopaminergic modulation of amygdala activity during emotion recognition in patients with Parkinson disease," Journal of Clinical Psychopharmacology, vol. 29, no. 6, pp. 548-554, 2009.

[203] C. Kelly, G. de Zubicaray, A. Di Martino et al., "L-dopa modulates functional connectivity in striatal cognitive and motor networks: a double-blind placebo-controlled study," Journal of Neuroscience, vol. 29, no. 22, pp. 7364-7378, 2009.

[204] P. Delaveau, P. Salgado-Pineda, B. Wicker, J. Micallef-Roll, and O. Blin, "Effect of levodopa on healthy volunteers' facial emotion perception: an fMRI study," Clinical Neuropharmacology, vol. 28, no. 6, pp. 255-261, 2005.

[205] P. Delaveau, P. Salgado-Pineda, J. Micallef-Roll, and O. Blin, "Amygdala activation modulated by levodopa during emotional recognition processing in healthy volunteers: a double-blind, placebo-controlled study," Journal of Clinical Psychopharmacology, vol. 27, no. 6, pp. 692-697, 2007.

[206] V. Voon, B. Reynolds, C. Brezing et al., "Impulsive choice and response in dopamine agonist-related impulse control behaviors," Psychopharmacology, vol. 207, no. 4, pp. 645-659, 2010 . 


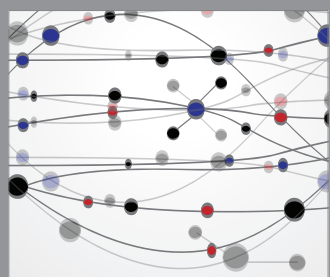

The Scientific World Journal
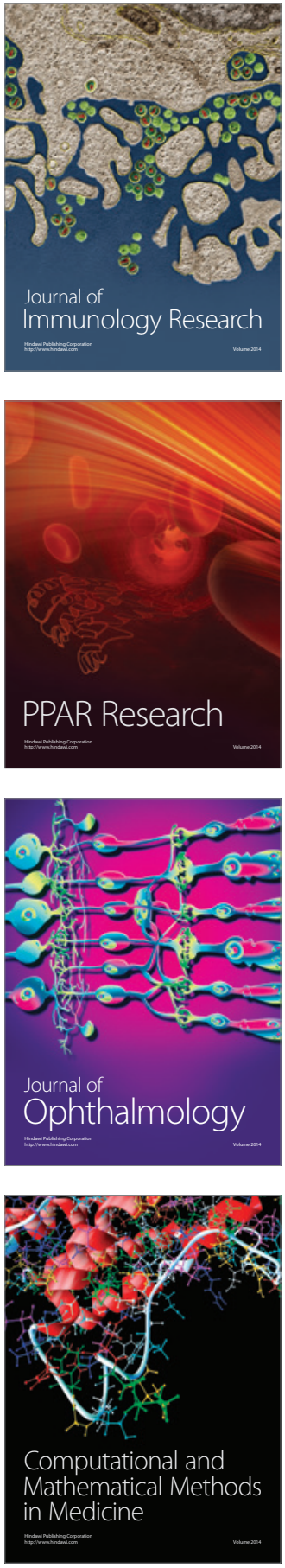

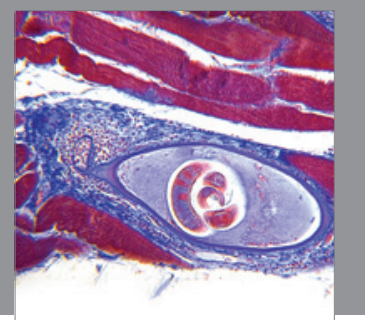

Gastroenterology

Research and Practice
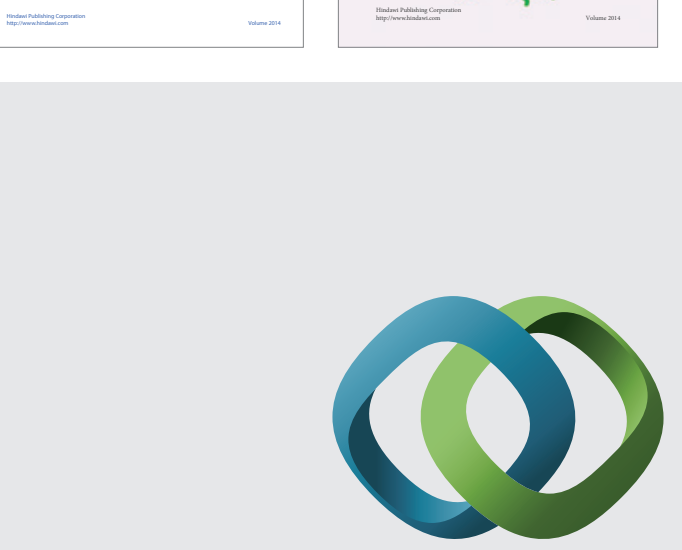

\section{Hindawi}

Submit your manuscripts at

http://www.hindawi.com
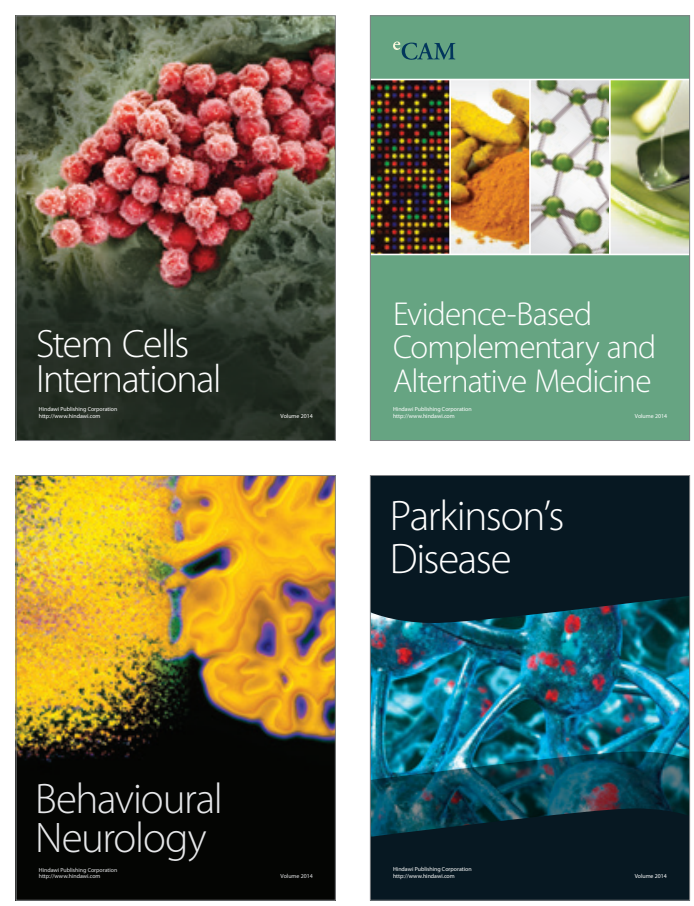

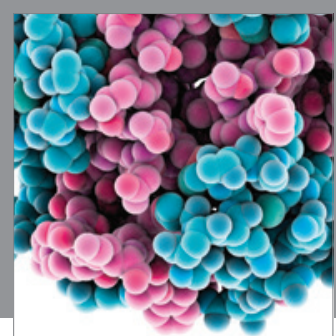

Journal of
Diabetes Research

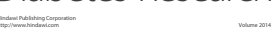

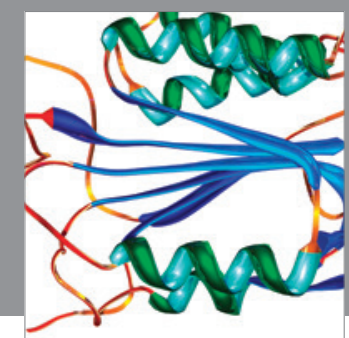

Disease Markers
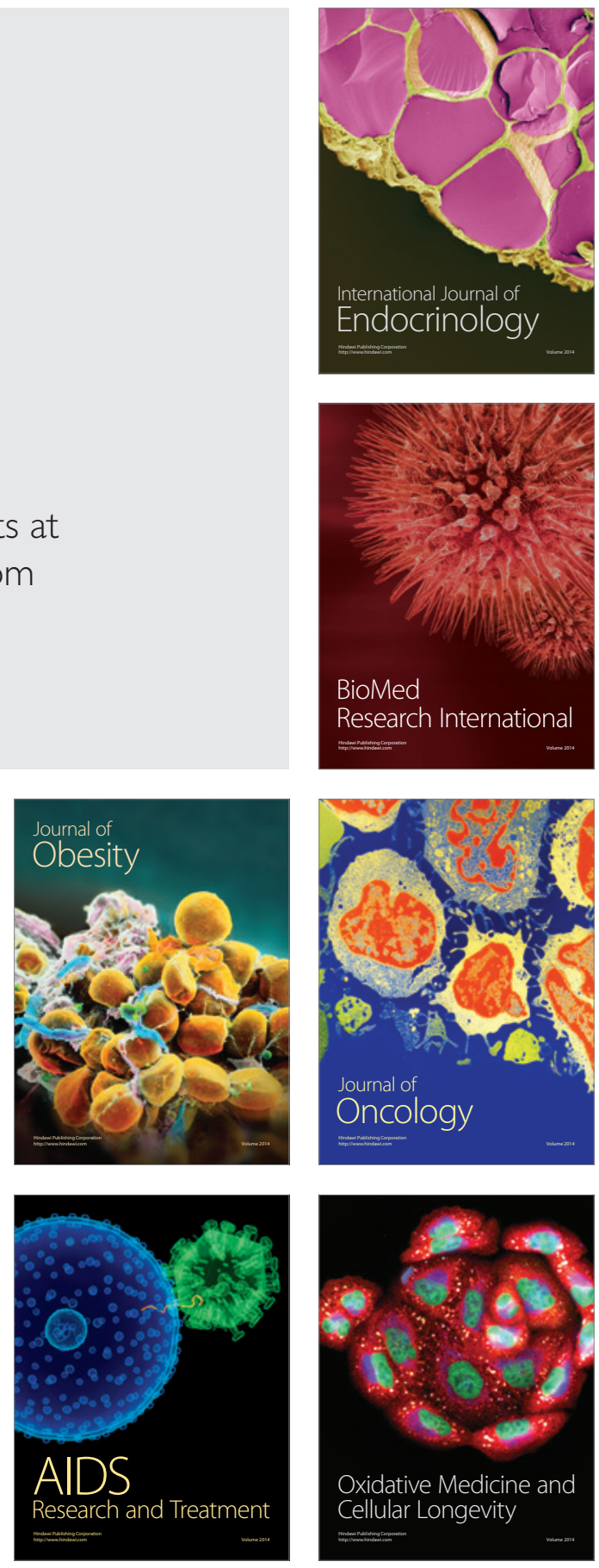